K. Sato, T. Watanabe, K. Yamamuro and M. Yamazato

Nagoya Math. J.

Vol. 141 (1996), 45-78

\title{
MULTIDIMENSIONAL PROCESS OF ORNSTEIN-UHLENBECK TYPE WITH NONDIAGONALIZABLE MATRIX IN LINEAR DRIFT TERMS
}

\author{
KEN-ITI SATO, TOSHIRO WATANABE, \\ KOUJI YAMAMURO AND MAKOTO YAMAZATO
}

\section{Introduction and results}

Let $\mathbf{R}^{d}$ be the $d$-dimensional Euclidean space where each point is expressed by a column vector. Let $|x|$ and $\langle x, y\rangle$ denote the norm and the inner product in $\mathbf{R}^{d}$. Let $Q=\left(Q_{j k}\right)$ be a real $d \times d$-matrix of which all eigenvalues have positive real parts. Let $\mathbf{X}$ be a process of Ornstein-Uhlenbeck type (OU type process) on $\mathbf{R}^{d}$ associated with a Lévy process $\left\{Z_{t}: t \geq 0\right\}$ and the matrix $Q$. Main purpose of this paper is to give a recurrence-transience criterion for the process $\mathbf{X}$ when $Q$ is a Jordan cell matrix and to compare it with the case when $Q$ is diagonalizable. Here by a Lévy process we mean a stochastically continuous process with stationary independent increments, starting at 0 . By saying that $Q$ is a Jordan cell matrix (with eigenvalue $\alpha$ ) we mean that

$$
Q_{j j}=\alpha \text { for } 1 \leq j \leq d, Q_{j, j+1}=1 \text {, for } 1 \leq j \leq d-1 \text {, and } Q_{j k}=0 \text { otherwise. }
$$

This paper continues the work [1], where a recurrence-transience criterion is established when $Q$ is diagonalizable. In one dimension the criterion is given by Shiga [5].

Precise definition of the process $\mathbf{X}$ by its infinitesimal generator is given in [1] and [3]. It is a Markov process $\left(\Omega, \mathscr{F}, \mathscr{F}_{t}, \mathbf{P}^{x}, X_{t}\right)$ on $\mathbf{R}^{d}$ such that the process $\left\{X_{t}: t \geq 0\right\}$ under the probability measure $\mathbf{P}^{x}$ is equivalent to the process $\left\{\bar{X}_{t}\right\}$ defined by

$$
\bar{X}_{t}=e^{-t Q} x+\int_{0}^{t} e^{-(t-r) Q} d Z_{r}
$$

Received February 22, 1994. 
where the stochastic integral with respect to the Lévy process is defined by stochastic convergence from integrals of simple functions. The process $\left\{\bar{X}_{t}\right\}$ of (1.1) is the unique solution of the equation

$$
\bar{X}_{t}=x+Z_{t}-\int_{0}^{t} Q \bar{X}_{r} d r
$$

This shows that $-Q$ is the coefficient matrix in the linear drift terms in the infinitesimal generator. We do not impose any restriction on the Lévy process $\left\{Z_{t}\right\}$ on $\mathbf{R}^{d}$ in this paper. Let $\rho$ and $B$ be the Lévy measure and the Gaussian covariance matrix of $\left\{Z_{t}\right\}$, respectively. That is, $\rho$ is a measure on $\mathbf{R}^{d}$ satisfying $\rho(\{0\})=0$ and $\int\left(|x|^{2} \wedge 1\right) \rho(d x)<\infty, B$ is a symmetric nonnegative-definite $d \times d$-matrix, and

$$
\begin{aligned}
& \mathbf{E} e^{i\left\langle z, Z_{t}\right\rangle}=\exp \left[-\frac{1}{2}\langle z, B z\rangle\right. \\
& \left.\quad+\int\left(e^{i\langle z, x\rangle}-1-i\langle z, x\rangle 1_{\{|x|<1\}}(x)\right) \rho(d x)+i\langle b, z\rangle\right],
\end{aligned}
$$

where $b$ is a $d$-vector and $1_{\{|x|<1\}}(x)$ is the indicator function of the unit disc.

Let $\left\{x_{t}\right\}$ be the dynamical system defined by

$$
\frac{d x_{t}}{d t}=-Q x_{t} \text { with } x_{0}=x
$$

that is $x_{t}=e^{-t Q} x$. As is seen from (1.1) and (1.2), the Markov process $\mathbf{X}$ is interpreted as the dynamical system with a time homogeneous random perturbation added independently of the past history and the present position. If $\left\{Z_{t}\right\}$ is Brownian, then $\mathbf{X}$ is the usual Ornstein-Uhlenbeck process and has a Gaussian limit distribution. In general, since $x_{t}$ tends to 0 as $t \rightarrow \infty$ (the origin is a sink), the process $\mathbf{X}$ has a limit distribution unless the frequency of big jumps of the process $\left\{Z_{t}\right\}$ is beyond a certain extent. A necessary and sufficient condition for $\mathbf{X}$ to have a limit distribution is that

$$
\int_{|x| \geq 1} \log |x| \rho(d x)<\infty
$$

(see [2], [3], and papers cited in [1]). A necessary and sufficient condition for $\mathbf{X}$ to be recurrent should be weaker than this.

From now on assume that $d \geq 2$. Let us denote 


$$
g(s ; x)=g\left(s ; x_{1}, \ldots, x_{d}\right)=x_{1}+x_{2} s+x_{3} \frac{s^{2}}{2 !}+\cdots+x_{d} \frac{s^{d-1}}{(d-1) !} .
$$

Then a main result in this paper is as follows. Our definition of recurrence and transience is given in [1].

THEOREM A. Suppose that $Q$ is a Jordan cell matrix with eigenvalue $\alpha>0$. Fix $c>0$ arbitrarily. Then $\mathbf{X}$ is recurrent if and only if

$$
\int_{0}^{1} \frac{d v}{v} \exp \left[\int_{v}^{1} \frac{d u}{u} \int_{|x| \geq c}\left(e^{-u^{\alpha}|g(\log u ; x)|}-1\right) \rho(d x)\right]=\infty .
$$

Note that the recurrence condition in the theorem above does not involve the measure $\rho$ on any compact set, nor $B$, nor $b$.

In Section 2 we will prove Theorem A. Our method of proof is Fourier-analytic as in [1] and [4]. The idea includes a way how to handle nonsymmetricity. In carrying through this method estimation of some integrals of elementary functions is crucial. It is here that we have to overcome difficulty caused by nondiagonalizability of $Q$. See Lemmas $2.1-2.4$.

In Section 3 we will show the following theorem, which gives another form to Theorem A and unifies it with the result of [1].

THEOREM B. Suppose that the eigenvalues of $Q$ are real and that there is an invertible matrix $R$ such that $R Q R^{-1}$ is either a diagonal matrix or a Jordan cell matrix. Fix $c>0$. Then, $\mathbf{X}$ is recurrent if and only if

$$
\int_{0}^{1} \frac{d v}{v} \exp \left[\int_{v}^{1} \frac{d u}{u} \int_{|x| \geq c}\left(e^{-\left|u^{Q} x\right|}-1\right) \rho(d x)\right]=\infty,
$$

where $u^{Q}=e^{(\log u) Q}$

When $Q$ is a general nondiagonalizable matrix, to find a recurrencetransience criterion is still an open problem.

In Sections 4 and 5 we restrict our attention to the case $d=2$. Let

$$
Q_{0}=\left(\begin{array}{cc}
\alpha & 0 \\
0 & \alpha
\end{array}\right), \quad Q_{1}=\left(\begin{array}{cc}
\alpha & 1 \\
0 & \alpha
\end{array}\right), \quad \alpha>0 .
$$

With a Lévy process $\left\{Z_{t}\right\}$ on $\mathbf{R}^{2}$ being fixed, we denote, for $j=0,1$, the OU type process on $\mathbf{R}^{2}$ associated with $\left\{Z_{t}\right\}$ and $Q_{j}$ by $\mathbf{X}_{j}$. We tackle the problems whether recurrence of $\mathbf{X}_{0}$ implies recurrence of $\mathbf{X}_{1}$ and whether the converse implication is true. It turns out that these problems are of delicate nature. The answers to both 
questions are negative. Thus the condition (1.6) on the Lévy measure $\rho$ with $Q=$ $Q_{0}$ is neither stronger nor weaker than the condition (1.6) with $Q=Q_{1}$. A probabilistically deeper fact is contained in the following theorem. The words increase and decrease are used in this paper in the wide sense allowing flatness. The restriction of $\rho$ to a set $E$ is denoted by $[\rho]_{E}$.

THEOREM C. (i) There exists a right-continuous positive function $h(r)$ on $[e, \infty)$, decreasing to 0 as $r \rightarrow \infty$, such that

$$
\begin{gathered}
\int_{0}^{1 / e} \frac{d v}{v} \exp \left[-\int_{e}^{1 / v} \frac{h(r)}{r} d r\right]=\infty, \\
\int_{0}^{1 / e} \frac{d v}{v} \exp \left[-\int_{e}^{1 / v} \frac{h(r)}{r}\left(1+\frac{1}{\log r}\right) d r\right]<\infty .
\end{gathered}
$$

(ii) Assume that the function $h(r)$ defined from the Lévy measure $\rho$ by

$$
h(r)=\frac{1}{\alpha} \int_{\left|x_{2}\right|>r} \rho(d x) \text { for } r \geq e
$$

satisfies (1.8) and (1.9). Fix $x_{0}=\left(\begin{array}{l}x_{01} \\ x_{02}\end{array}\right)$ with $x_{02} \neq 0$ and let

$$
E_{j}=\left\{x=u^{Q_{j}} x_{0}: u>0\right\} \text { for } j=0,1 .
$$

If, for some $c>0,[\rho]_{\{|x|>c\}}$ is concentrated on $E_{0}$, then $\mathbf{X}_{0}$ is recurrent and $\mathbf{X}_{1}$ is transient. If, for some $c>0,[\rho]_{\{|x|>c\}}$ is concentrated on $E_{1}$, then $\mathbf{X}_{0}$ is transient and $\mathbf{X}_{1}$ is recurrent.

Note that the set $E_{j}$ is identical with the trajectory of the dynamical system

$$
\frac{d x_{t}}{d t}=-Q_{j} x_{t}, \quad-\infty<t<\infty .
$$

It is remarkable that concentration of the Lévy measure on $E_{j}$ is relevant to recurrence of the process $\mathbf{X}_{j}$, although any jump from $x$ in this case is to $x+E_{j}$, not to the trajectory (of the dynamical system) that $x$ belongs to.

Proof of Theorem C will be given in Section 4. The last theorem, which is to be proved in Section 5 , shows that, under a mild additional condition, recurrence of $\mathbf{X}_{0}$ is equivalent to recurrence of $\mathbf{X}_{1}$.

THEOREM D. Suppose that 


$$
\int_{|x|>e} \log \log |x| \rho(d x)<\infty
$$

Then $\mathbf{X}_{0}$ is recurrent if and only if $\mathbf{X}_{1}$ is recurrent.

The examples in Section 5 of [1] satisfy the condition (1.11). They include both recurrent and transient cases.

\section{Recurrence criterion in the case where $Q$ is a Jordan cell matrix}

Proof of Theorem A needs several lemmas of analytic nature.

Lemma 2.1. Let $f(s)$ be a polynomial with real coefficients with $\operatorname{deg} f \leq n$. Let $\alpha>0$ and $0<M<N$. Then

$$
\left|\int_{M}^{N} \sin \left(u^{\alpha} f(\log u)\right) \frac{d u}{u}\right| \leq \frac{K_{1}}{\alpha}
$$

and

$$
\left|\int_{M}^{N}\left[\cos \left(u^{\alpha} f(\log u)\right)-e^{-\left|u^{\alpha} f(\log u)\right|}\right] \frac{d u}{u}\right| \leq \frac{K_{2}}{\alpha},
$$

where $K_{1}$ and $K_{2}$ are constants which depend only on $n$.

Proof. By substitution $u^{\alpha}=v$, we see that it suffices to prove the lemma for $\alpha=1$. Let $c$ be the coefficient of $s^{n}$ in $f(s)$. Since the integrals in (2.1) and (2.2) are continuous in $c$, we may assume that $c \neq 0$. Further, we may assume that $c>$ 0 . By substitution $c u=v$, we get

$$
\int_{M}^{N} \sin (u f(\log u)) \frac{d u}{u}=\int_{c M}^{c N} \sin (v \tilde{f}(\log v)) \frac{d v}{v},
$$

where $\tilde{f}$ is a polynomial of degree $n$ with coefficient 1 in the highest term, and similarly for the integral in (2.2). Henceforth let

$$
f(s)=\prod_{k=1}^{p}\left(s-a_{k}\right) \cdot \prod_{k=p+1}^{p+q}\left(\left(s-a_{k}\right)^{2}+c_{k}^{2}\right),
$$

where $p \geq 0, q \geq 0, p+2 q=n$, and $a_{k}$ and $c_{k}$ are real, $c_{k}>0$. Denote by $I(M, N)$ and $J(M, N)$ the integrals in (2.1) and (2.2), respectively, with $\alpha=1$ and $f(s)$ being of (2.3). In order to prove the lemma, it is enough to show that $|I(M, N)|$ 
$\leq K_{1}(p, q)$ and $|J(M, N)| \leq K_{2}(p, q)$, where $K_{1}(p, q)$ and $K_{2}(p, q)$ depend only on $p$ and $q$. Let $h(u)=u f(\log u)$. If $h^{\prime}(u) \neq 0$ on the interval $(M, N)$, then substitution $v=h(u)$ gives

$$
I(M, N)=\int_{h(M)}^{h(N)} \frac{\sin v}{u h^{\prime}(u)} d v, \quad J(M, N)=\int_{h(M)}^{h(N)} \frac{\cos v-e^{-|v|}}{u h^{\prime}(u)} d v
$$

Let $s=\log u$. We note that

$$
\begin{aligned}
\frac{f^{\prime}(s)}{f(s)}= & \sum_{k=1}^{p} \frac{1}{s-a_{k}}+\sum_{k=p+1}^{p+q} \frac{2\left(s-a_{k}\right)}{\left(s-a_{k}\right)^{2}+c_{k}^{2}}, \\
\frac{f^{\prime \prime}(s)}{f(s)}= & \left(\frac{f^{\prime}(s)}{f(s)}\right)^{2}-\sum_{k=1}^{p} \frac{1}{\left(s-a_{k}\right)^{2}} \\
& -\sum_{k=p+1}^{p+q} \frac{4\left(s-a_{k}\right)^{2}}{\left(\left(s-a_{k}\right)^{2}+c_{k}^{2}\right)^{2}}+\sum_{k=p+1}^{p+q} \frac{2}{\left(s-a_{k}\right)^{2}+c_{k}^{2}},
\end{aligned}
$$

$$
\begin{aligned}
& u h^{\prime}(u)=h(u)\left(1+\frac{f^{\prime}(s)}{f(s)}\right) \\
& \frac{d\left(u h^{\prime}(u)\right)}{d v}=\frac{d\left(u h^{\prime}(u)\right)}{d u} \frac{d u}{d v}=\left(1+2 \frac{f^{\prime}(s)}{f(s)}+\frac{f^{\prime \prime}(s)}{f(s)}\right)\left(1+\frac{f^{\prime}(s)}{f(s)}\right)^{-1} .
\end{aligned}
$$

Choose $\eta>0$ so that

$$
2 \frac{n}{\eta}+\frac{n^{2}+p+6 q}{\eta^{2}}<1
$$

If $s \notin \cup_{k=1}^{p+q}\left(a_{k}-\eta, a_{k}+\eta\right)$, then it follows from (2.5) and (2.6) that

$$
\left|\frac{f^{\prime}(s)}{f(s)}\right| \leq \frac{n}{\eta}<\frac{1}{2},\left|2 \frac{f^{\prime}(s)}{f(s)}+\frac{f^{\prime \prime}(s)}{f(s)}\right| \leq 2 \frac{n}{\eta}+\frac{n^{2}+p+6 q}{\eta^{2}}<1 .
$$

Let $E=(0, \infty) \backslash \cup_{k=1}^{p+q}\left[e^{a_{k}-\eta}, e^{a_{k}+\eta}\right]$ and let $\left(A_{1}, B_{1}\right), \ldots,\left(A_{m-1}, B_{m-1}\right),\left(A_{m}, B_{m}\right)$ with $B_{m}=\infty$ be the connected components of $E$. We have $m \leq p+q+1$. Consider two cases:

Case 1. $M, N \in\left[e^{a_{k}-\eta}, e^{a_{k}+\eta}\right]$ for some $k$.

Case 2. $M, N \in\left(A_{l}, B_{l}\right)$ for some $l$.

In Case 1 the estimate is easy. Namely

$$
|I(M, N)| \leq \int_{\exp \left(a_{k}-\eta\right)}^{\exp \left(a_{k}+\eta\right)} \frac{d u}{u}=2 \eta
$$

and, similarly, $|J(M, N)| \leq 4 \eta$. 
Let us consider Case 2. No zeros of $h(u)$ exist in $\left(A_{l}, B_{l}\right)$. First we assume that $h(u)>0$ on $\left(A_{l}, B_{l}\right)$. By $(2.5)-(2.10), h^{\prime}(u)$ is positive on $\left(A_{l}, B_{l}\right)$ and $\frac{d}{d v}\left(u h^{\prime}(u)\right)$ is positive for $v \in\left(h\left(A_{l}\right), h\left(B_{l}\right)\right)$. Hence we have

$$
\left|I\left(A_{l}, N\right)\right|=\left|\int_{h\left(A_{l}\right)}^{h(N)} \frac{\sin v}{u h^{\prime}(u)} d v\right| \leq\left|\int_{h\left(A_{l}\right)}^{((j+1) \pi) \wedge h\left(B_{l}\right)}\right|+\left|\int_{((j+1) \pi) \wedge h\left(B_{l}\right)}^{\left(h\left(A_{l}\right)+\pi\right) \wedge h\left(B_{l}\right)}\right|,
$$

where $j$ is the nonnegative integer determined by $j \pi \leq h\left(A_{l}\right)<(j+1) \pi$. Therefore, using $u h^{\prime}(u)>v / 2$, we obtain

$$
\left|I\left(A_{l}, N\right)\right| \leq 2\left|\int_{j \pi}^{(j+1) \pi} \frac{\sin v}{v} d v\right| \leq 2 \int_{0}^{\pi} \frac{\sin v}{v} d v .
$$

Hence

$$
|I(M, N)|=\left|I\left(A_{l}, N\right)-I\left(A_{l}, M\right)\right| \leq 4 \int_{0}^{\pi} \frac{\sin v}{v} d v
$$

If $h(N) \leq \pi / 2$, then

$$
\left|J\left(A_{l}, N\right)\right| \leq \int_{h\left(A_{l}\right)}^{h(N)} \frac{\left|\cos v-e^{-v}\right|}{u h^{\prime}(u)} d v \leq 2 \int_{0}^{\pi / 2} \frac{\left|\cos v-e^{-v}\right|}{v} d v .
$$

If $h\left(A_{l}\right) \leq \pi / 2<h(N)$, then

$$
\begin{aligned}
\left|J\left(A_{l}, N\right)\right| & \leq \int_{h\left(A_{l}\right)}^{\pi / 2} \frac{\left|\cos v-e^{-v}\right|}{u h^{\prime}(u)} d v+\left|\int_{\pi / 2}^{h(N)} \frac{\cos v}{u h^{\prime}(u)} d v\right|+\int_{\pi / 2}^{h(N)} \frac{e^{-v}}{u h^{\prime}(u)} d v \\
& \leq 2 \int_{0}^{\pi / 2} \frac{\left|\cos v-e^{-v}\right|}{v} d v+2\left|\int_{\pi / 2}^{3 \pi / 2} \frac{\cos v}{v} d v\right|+2 \int_{\pi / 2}^{\infty} \frac{e^{-v}}{v} d v
\end{aligned}
$$

If $\pi / 2<h\left(A_{l}\right)$, then, using the integer $j$ determined by $\pi / 2+j \pi \leq h\left(A_{l}\right)<\pi / 2$ $+(j+1) \pi$, we have

$$
\begin{aligned}
\left|J\left(A_{l}, N\right)\right| & \leq\left|\int_{h\left(A_{l}\right)}^{h(N)} \frac{\cos v}{u h^{\prime}(u)} d v\right|+\int_{h\left(A_{l}\right)}^{h(N)} \frac{e^{-v}}{u h^{\prime}(u)} d v \\
& \leq 2\left|\int_{\pi / 2+j \pi}^{\pi / 2+(j+1) \pi} \frac{\cos v}{v} d v\right|+2 \int_{\pi / 2+j \pi}^{\infty} \frac{e^{-v}}{v} d v \\
& \leq 2\left|\int_{\pi / 2}^{3 \pi / 2} \frac{\cos v}{v} d v\right|+2 \int_{\pi / 2}^{\infty} \frac{e^{-v}}{v} d v .
\end{aligned}
$$

Hence $|J(M, N)|$ is bounded by an absolute constant. In the case where $h(u)<0$ on $\left(A_{l}, B_{l}\right)$, we have $h^{\prime}(u)<0$ on $\left(A_{l}, B_{l}\right), 0>h(M)>h(N)$, and $-u h^{\prime}(u)$ is a decreasing function of $v$. Hence, also in this case, the same discussion applies to 
the majorization of $\left|I\left(A_{l}, N\right)\right|$ and $\left|J\left(A_{l}, N\right)\right|$. Thus $|I(M, N)|$ and $|J(M, N)|$ are bounded by absolute constants in Case 2 . As the integrals in general case are sums of the integrals of Cases 1 and 2, the proof is complete.

Lemma 2.2. Let $f(s)$ be a polynomial with complex coefficients with $\operatorname{deg} f \leq n$. Let $\alpha>0$ and $0<a<b$. Then

$$
\int_{0}^{\infty}\left(e^{-a u^{\alpha}|f(\log u)|}-e^{-b u^{\alpha}|f(\log u)|}\right) \frac{d u}{u} \leq \frac{1}{\alpha}\left(K_{3} \log \frac{b}{a}+K_{4}\right)
$$

with constants $K_{3}$ and $K_{4}$ which depend only on $n$.

Proof. Substituting $u^{2 \alpha}=v$, we see that it suffices to give the proof for $\alpha=$ $1 / 2$. Further we may and do assume that the coefficient of $s^{n}$ in $f(s)$ is 1 . Let $F(s)=|f(s)|^{2}$. Then, for real $s, F(s)$ equals a polynomial of degree $2 n$ with real coefficients with coefficient 1 in the highest term. Factorize it as in the right-hand side of (2.3) with $p+2 q=2 n$. Let $v=h(u)=u F(\log u)$. Choose $\eta>0$ satisfying (2.9) with $2 n$ in place of $n$. Consider

$$
I=\int_{A}^{B}\left(e^{-a u^{1 / 2} F(\log u)^{1 / 2}}-e^{-b u^{1 / 2} F(\log u)^{1 / 2}}\right) \frac{d u}{u} .
$$

If $A=e^{a_{k}-\eta}$ and $B=e^{a_{k}+\eta}$, then $I \leq 2 \eta$. Let $A=A_{l}$ and $B=B_{l}$, where $A_{l}$ and $B_{l}$ are defined as in the proof of Lemma 2.1. Then, $h(u)>0$ and $u h^{\prime}(u)>h(u) / 2$ on $(A, B)$. Hence

$$
I=\int_{h(A)}^{h(B)} \frac{e^{-a v^{1 / 2}}-e^{-b v^{1 / 2}}}{u h^{\prime}(u)} d v \leq 2 \int_{0}^{\infty} \frac{e^{-a v^{1 / 2}}-e^{-b v^{1 / 2}}}{v} d v=4 \log \frac{b}{a} .
$$

Summing up these estimates, we get (2.11).

Lemma 2.3. Let $c, a_{1}, \ldots, a_{n}, b_{1}, \ldots, b_{n}$ be complex numbers and let

$$
f(s)=c \prod_{k=1}^{n}\left(s-a_{k}\right), \quad \tilde{f}(s)=c \prod_{k=1}^{n}\left(s-b_{k}\right) .
$$

Then

$$
\begin{aligned}
& \int_{0}^{\infty}\left|e^{-u^{\alpha}|f(\log u)|}-e^{-u^{\alpha}|\tilde{f}(\log u)|}\right| \frac{d u}{u} \\
& \quad \leq \frac{2 K_{3}}{\alpha} \sum_{k=1}^{n} \log \left(1+\left|a_{k}-b_{k}\right|\right)+\frac{2 n}{\alpha} K_{4}+4 n,
\end{aligned}
$$


where $K_{3}$ and $K_{4}$ are the constants in Lemma 2.2 .

Proof. Let $h(s)=c \Pi_{k=2}^{n}\left(s-a_{k}\right)$ and consider the integral in (2.12) with $\left(s-b_{1}\right) h(s)$ in place of $\tilde{f}(s)$. Let

$$
E_{1}=\left\{u>0:\left|\log u-a_{1}\right| \leq\left|\log u-b_{1}\right|\right\},
$$

and let $E_{2}$ be the complement of $E_{1}$ in $(0, \infty)$. Then

$$
\begin{aligned}
& \int_{E_{1}}\left(e^{-u^{\alpha}\left|\log u-a_{1}\right||h(\log u)|}-e^{-u^{\alpha}\left|\log u-b_{1}\right||h(\log u)|}\right) \frac{d u}{u} \\
& \leq \int_{0}^{\infty} e^{-u^{\alpha}\left|\log u-a_{1}\right||h(\log u)|}\left(1-e^{-u^{\alpha}\left|a_{1}-b_{1}\right||h(\log u)|}\right) \frac{d u}{u} .
\end{aligned}
$$

Partition the integral over $(0, \infty)$ into that over $F=\left[e^{\operatorname{Re} a_{1}-1}, e^{\mathrm{Re} a_{1}+1}\right]$ and that over $(0, \infty) \backslash F$. The former is clearly less than 2 . The latter is majorized by

$$
\int_{0}^{\infty}\left(e^{-u^{\alpha}|h(\log u)|}-e^{-u^{\alpha}\left(1+\left|a_{1}-b_{1}\right|\right)|h(\log u)|}\right) \frac{d u}{u},
$$

which is not greater than $\alpha^{-1} K_{3} \log \left(1+\left|a_{1}-b_{1}\right|\right)+\alpha^{-1} K_{4}$ by Lemma 2.2. Similarly the integral over $E_{2}$ is estimated. Repeating this procedure $n$ times, we obtain (2.12).

Lemma 2.4. For $k=1, \ldots, d$ let $x_{k}$ and $z_{k}$ be real numbers with $\left|z_{k}\right| \leq 1$. Suppose that $z_{1} \neq 0$. Let $g(s)$ be the function $g(s ; x)$ of $(1.4)$. Let

$$
\begin{aligned}
& f(s)=\sum_{k=1}^{d} z_{k} g^{(k-1)}(s), \\
& G(s)=\sum_{k=1}^{d}\left|g^{(k-1)}(s)\right|,
\end{aligned}
$$

where $g^{(k-1)}(s)$ is the $(k-1)$-th derivative of $g(s)$. Then, for any $\alpha>0$,

$$
\begin{aligned}
& \int_{0}^{\infty}\left|e^{-u^{\alpha}|f(\log u)|}-e^{-u^{\alpha}|g(\log u)|}\right| \frac{d u}{u} \leq \frac{1}{\alpha}\left(K_{5} \log \frac{1}{\left|z_{1}\right|}+K_{6}\right)+K_{7}, \\
& \int_{0}^{\infty}\left|e^{-u^{\alpha}|f(\log u)|}-e^{-u^{\alpha} G(\log u)}\right| \frac{d u}{u} \leq \frac{1}{\alpha}\left(K_{5} \log \frac{1}{\left|z_{1}\right|}+K_{8}\right)+K_{9}
\end{aligned}
$$

where $K_{5}, \ldots, K_{9}$ are constants that depend only on $d$. 
Proof. We may and do assume that $x_{d} \neq 0$. Define $\tilde{f}(s)$ and $h(s)$ by $\tilde{f}(s)=$ $\frac{1}{z_{1}} f(s)=g(s)+h(s)$. Let

$$
\begin{aligned}
& I_{1}=\int_{0}^{\infty}\left(e^{-u^{\alpha}|f(\log u)|}-e^{-u^{\alpha}|\tilde{f}(\log u)|}\right) \frac{d u}{u}, \\
& I_{2}=\int_{0}^{\infty}\left|e^{-u^{\alpha}|\tilde{f}(\log u)|}-e^{-u^{\alpha}|g(\log u)|}\right| \frac{d u}{u} .
\end{aligned}
$$

Then the integral in (2.15) is bounded by $I_{1}+I_{2}$. By Lemma 2.2,

$$
I_{1} \leq \frac{1}{\alpha}\left(K_{3} \log \frac{1}{\left|z_{1}\right|}+K_{4}\right)
$$

In order to estimate $I_{2}$ by using Lemma 2.3, factorize $g(s)$ as

$$
g(s)=\frac{x_{d}}{(d-1) !} \prod_{k=1}^{d-1}\left(s-a_{k}\right)
$$

where $a_{1}, \ldots, a_{d-1}$ are in the complex plane $\mathbf{C}$ and depend on $x_{1}, \ldots, x_{d}$. Denote $A$ $=\max _{2 \leq k \leq d}\left|z_{k} / z_{1}\right|$. We claim that

$$
\tilde{f}(s)=\frac{x_{d}}{(d-1) !} \prod_{k=1}^{d-1}\left(s-b_{k}\right),
$$

with some $b_{1}, \ldots, b_{d-1} \in \mathbf{C}$ satisfying

$$
\left|a_{k}-b_{k}\right| \leq K_{10}(A+1) \text { for } k=1, \ldots, d-1,
$$

where $K_{10}$ is a constant depending only on $d$. Let

$$
D=\bigcup_{k=1}^{d-1}\left\{s \in \mathbf{C}:\left|s-a_{k}\right|<\eta\right\}, \quad \eta=(d-1) !(d-1) A+1 .
$$

Then, for $s$ on the boundary $\partial D$ of $D$,

$$
\begin{aligned}
\left|\frac{h(s)}{g(s)}\right|= & \mid \frac{z_{2}}{z_{1}} \sum_{k_{1}} \frac{1}{s-a_{k_{1}}}+2 ! \frac{z_{3}}{z_{1}} \sum_{k_{1}<k_{2}} \frac{1}{\left(s-a_{k_{1}}\right)\left(s-a_{k_{2}}\right)} \\
& +\cdots+(d-1) ! \frac{z_{d}}{z_{1}} \frac{1}{\left(s-a_{1}\right) \cdots\left(s-a_{d-1}\right)} \mid \\
\leq & A\left((d-1) \frac{1}{\eta}+\left(\begin{array}{c}
d-1 \\
2
\end{array}\right) \frac{2 !}{\eta^{2}}+\left(\begin{array}{c}
d-1 \\
3
\end{array}\right) \frac{3 !}{\eta^{3}}+\cdots+\frac{(d-1) !}{\eta^{d-1}}\right) \\
\leq & \frac{(d-1) !(d-1) A}{\eta}<1 .
\end{aligned}
$$

Let $D_{1}$ be a connected component of $D$. Suppose that the number of zeros (with 
multiplicity counted) of $g(s)$ in $D_{1}$ is $m$. Then $D_{1}=\cup_{j=1}^{m}\left\{s \in \mathbf{C}:\left|s-a_{k_{j}}\right|\right.$ $<\eta\}$ with some $k_{1}, \ldots, k_{m}$ and $\partial D_{1} \subset \partial D$. Hence, by Rouché's theorem, $\tilde{f}(s)$ has exactly $m$ zeros in $D_{1}$. Denote them by $b_{k_{1}}, \ldots, b_{k_{m}}$. Then, for $j=1, \ldots, m$,

$$
\left|a_{k_{j}}-b_{k_{j}}\right|<(2 m-1) \eta \leq(2 d-3) \eta \leq K_{10}(A+1)
$$

with some $K_{10}$. Making this procedure to all connected components, we get (2.18) and (2.19). Since $\left|z_{k}\right| \leq 1$ for all $k$, we have $A+1 \leq 2 /\left|z_{1}\right|$. Now Lemma 2.3 yields

$$
I_{2} \leq \frac{2 K_{3}}{\alpha}(d-1) \log \left(1+\frac{2 K_{10}}{\left|z_{1}\right|}\right)+\frac{2 K_{4}}{\alpha}(d-1)+4(d-1) .
$$

Hence (2.15) is proved.

In order to get (2.16) from (2.15), it is enough to show that

$$
\int_{0}^{\infty}\left(e^{-u^{\alpha}|g(\log u)|}-e^{-u^{\alpha} G(\log u)}\right) \frac{d u}{u} \leq \frac{1}{\alpha} K_{11}+K_{12}
$$

with constants $K_{11}$ and $K_{12}$ depending only on $d$. Let $I_{3}$ be the integral in (2.20). Using $a_{k}$ in (2.17), let $E=\cap_{k=1}^{d-1}\left\{s \in \mathbf{C}:\left|s-a_{k}\right| \geq 1\right\}$. By the same estimate as above, we get

$$
\frac{\left|g^{\prime}(s)\right|+\cdots+\left|g^{(d-1)}(s)\right|}{|g(s)|} \leq(d-1) !(d-1) \text { for } s \in E .
$$

Therefore, letting $E^{\prime}=\{u>0: \log u \in E\}$, we have

$$
\begin{aligned}
I_{3} & \leq \sum_{k=1}^{d-1} \int_{\exp \left(\operatorname{Re} a_{k}-1\right)}^{\exp \left(\operatorname{Re} a_{k}+1\right)} \frac{d u}{u}+\int_{E^{\prime}}\left(e^{-u^{\alpha}|g(\log u)|}-e^{-u^{\alpha} G(\log u)}\right) \frac{d u}{u} \\
& \leq 2(d-1)+\int_{0}^{\infty}\left(e^{-u^{\alpha}|g(\log u)|}-e^{-K_{13} u^{\alpha}|g(\log u)|}\right) \frac{d u}{u} \\
& \leq 2(d-1)+\frac{1}{\alpha}\left(K_{3} \log K_{13}+K_{4}\right)
\end{aligned}
$$

with $K_{13}=1+(d-1) !(d-1)$. Here we used Lemma 2.2. Hence we have (2.20), completing the proof.

Now we can show that (1.5) is a recurrence criterion.

Proof of Theorem A. Let $\mathbf{X}$ be the OU type process associated with $\left\{Z_{t}\right\}$ and $Q$ of Theorem A. For $c>0$ denote by $\rho^{c}$ the restriction of the Lévy measure $\rho$ of $\left\{Z_{t}\right\}$ to the set $\{x:|x| \geq c\}$, and let $\left\{Z_{t}^{c}\right\}$ be the compound Poisson process hav- 
ing $\rho^{c}$ as its Lévy measure. Let $\mathbf{X}^{c}$ be the OU type process associated with $\left\{Z_{t}^{c}\right\}$ and $Q$. Let $p_{t}(x, E)$ and $p_{t}^{c}(x, E)$ be the transition probabilities of $\mathbf{X}$ and $\mathbf{X}^{c}$, respectively. Let $p_{t}(x, z)$ and $p_{t}^{c}(x, z)$ be their characteristic functions. Shiga [5] shows that transience of $\mathbf{X}$ is equivalent to that, for any $x$ and any compact set E,

$$
\int_{0}^{\infty} p_{t}(x, E) d t<\infty
$$

It is equivalent also to that there is a point $x$ such that, for any compact set $E$, (2.21) holds.

First let us notice that, for any $c^{\prime}>c>0$,

$$
\int_{v}^{1} \frac{d u}{u} \int_{c \leq|x|<c^{\prime}}\left(1-e^{-u^{\alpha}|g(\log u ; x)|}\right) \rho(d x)
$$

is bounded in $v \in(0,1)$. In fact, it is increasing as $v \downarrow 0$ and, for any $\varepsilon \in(0, \alpha)$, there is a constant $K$ such that

$$
\begin{gathered}
\int_{0}^{1} \frac{d u}{u} \int_{c \leq|x|<c^{\prime}}\left(1-e^{-u^{\alpha}|g(\log u ; x)|}\right) \rho(d x) \leq \int_{0}^{1} \frac{d u}{u} \int_{c \leq|x|<c^{\prime}}\left(1-e^{-K u^{\alpha-\varepsilon}|x|}\right) \rho(d x) \\
\quad \leq \frac{1}{\alpha-\varepsilon} \int_{c \leq|x|<c^{\prime}} \rho(d x) \int_{0}^{K c^{\prime}}\left(1-e^{-u}\right) \frac{d u}{u} .
\end{gathered}
$$

Therefore, if

$$
\int_{0}^{1} \frac{d u}{v} \exp \left[\int_{v}^{1} \frac{d u}{u} \int_{|x| \geq c}\left(e^{-u^{\alpha}|g(\log u ; x)|}-1\right) \rho(d x)\right]<\infty
$$

for some $c>0$, then it is true for any $c^{\prime}>0$ in place of $c$.

Suppose that $\mathbf{X}$ is transient. Let us prove (2.22). Transience of $\mathbf{X}$ implies transience of $\mathbf{X}^{c}$ for every $c>0$ ([5] p. 439 and [1] Lemma 2.1). Let $h(x)=$ $\Pi_{j=1}^{d}\left(\left(1-\left|x_{j}\right|\right) \vee 0\right)$. Let $\hat{h}(z)=\int e^{i\langle z, x\rangle} h(x) d x$, the Fourier transform of $h$. Then

$$
\hat{h}(z)=\prod_{j=1}^{d} 4 z_{j}^{-2} \sin ^{2}\left(2^{-1} z_{j}\right), \quad h(x)=(2 \pi)^{-d} \int e^{-i\langle z, x\rangle} \hat{h}(z) d z .
$$

Hence

$$
\infty>\int_{0}^{\infty} d t \int p_{t}^{c}(0, d x) h(x)=(2 \pi)^{-d} \int_{0}^{\infty} d t \int \hat{h}(z) p_{t}^{c}(0, z) d z
$$




$$
=(2 \pi)^{-d} \int_{0}^{\infty} d t \int \hat{h}(z) \operatorname{Re} \hat{p}_{t}^{c}(0, z) d z
$$

It follows from (1.1) that

$$
\hat{p}_{t}^{c}(0, z)=\exp \left[\int_{0}^{t} d r \int\left(e^{i\left\langle z, e^{-r Q} x\right\rangle}-1\right) \rho^{c}(d x)\right]
$$

(see [3]). Hence

$$
\operatorname{Re} p_{t}^{c}(0, z)=(\cos F(t, z))(\exp G(t, z)),
$$

where

$$
\begin{aligned}
& F(t, z)=\int_{0}^{t} d r \int \sin \left\langle z, e^{-r Q} x\right\rangle \rho^{c}(d x), \\
& G(t, z)=\int_{0}^{t} d r \int\left(\cos \left\langle z, e^{-r Q} x\right\rangle-1\right) \rho^{c}(d x) .
\end{aligned}
$$

Let $g(s ; x)$ be the function of $(1.4)$ and let $g^{(k)}(s ; x)$ be its $k$-th derivative in $s$. Since $Q$ is a Jordan cell matrix with eigenvalue $\alpha$, we have $u^{Q} x=\left(y_{j}\right)_{1 \leq j \leq d}$ with

$$
y_{1}=u^{\alpha} g(\log u ; x), y_{2}=u^{\alpha} g^{\prime}(\log u ; x), \cdots, y_{d}=u^{\alpha} g^{(d-1)}(\log u ; x) \text {. }
$$

Thus

$$
|F(t, z)|=\left|\int_{e^{-t}}^{1} \frac{d u}{u} \int \sin \left\langle z, u^{Q} x\right\rangle \rho^{\mathrm{c}}(d x)\right| \leq \frac{K_{1}}{\alpha} \rho^{\mathrm{c}}\left(\mathbf{R}^{d}\right)
$$

by Lemma 2.1. Choose $c$ so large that $\alpha^{-1} K_{1} \rho^{c}\left(\mathbf{R}^{d}\right)<\pi / 4$. Then $\cos F(t, z) \geq$ $1 / \sqrt{2}$ and, therefore,

$$
\int \hat{h}(z) d z \int_{0}^{\infty} \exp G(t, z) d t<\infty
$$

Let $U(z)=\int_{0}^{\infty} \exp G(t, z) d t$. Then $U(z)$ is finite for some (in fact almost every) $z=\left(z_{j}\right)_{1 \leq j \leq d}$ satisfying $\left|z_{j}\right| \leq 1$ and $z_{1} \neq 0$. By $e^{-r}=u$ and $e^{-t}=v$ we get

$$
U(z)=\int_{0}^{1} \frac{d v}{v} \exp \left[\int_{v}^{1} \frac{d u}{u} \int\left(\cos \left\langle z, u^{Q} x\right\rangle-1\right) \rho^{c}(d x)\right] .
$$

By virtue of Lemmas 2.1 and 2.4,

$$
U(z)=\int_{0}^{1} \frac{d v}{v} \exp \left[\int \rho ^ { c } ( d x ) \left\{\int_{v}^{1}\left(\cos \left\langle z, u^{Q} x\right\rangle-e^{-\left|\left\langle z, u^{Q} x\right\rangle\right|}\right) \frac{d u}{u}\right.\right.
$$




$$
\begin{aligned}
& \left.\left.+\int_{v}^{1}\left(e^{-\left|\left\langle z, u^{Q} x\right\rangle\right|}-e^{-u^{\alpha}|g(\log u ; x)|}\right) \frac{d u}{u}+\int_{v}^{1}\left(e^{-u^{\alpha}|g(\log u ; x)|}-1\right) \frac{d u}{u}\right\}\right] \\
\geq & C_{1} \int_{0}^{1} \frac{d v}{v} \exp \left[\int \rho^{c}(d x) \int_{v}^{1}\left(e^{-u^{\alpha}|g(\log u ; x)|}-1\right) \frac{d u}{u}\right],
\end{aligned}
$$

where

$$
C_{1}=\exp \left[-\rho^{c}\left(\mathbf{R}^{d}\right)\left(\frac{K_{2}}{\alpha}+\frac{K_{5}}{\alpha} \log \frac{1}{\left|z_{1}\right|}+\frac{K_{6}}{\alpha}+K_{7}\right)\right]
$$

Hence we get (2.22).

Conversely, suppose that (2.22) holds. We will prove transience of $\mathbf{X}$. Use $h_{a}(x)=\Pi_{j=1}^{d}\left(\left(a-\left|x_{j}\right|\right) \vee 0\right)$ with $a>0$. Its Fourier transform is $\hat{h}_{a}(z)=$ $\Pi_{j=1}^{d} 4 z_{j}^{-2} \sin ^{2}\left(2^{-1} a z_{j}\right)$. Hence, in order to show transience, it suffices to prove

$$
\int_{0}^{\infty} d t \int p_{t}(0, d x) \hat{h}_{a}(x)<\infty
$$

for all small $a>0$. But

$$
\int p_{t}(0, d x) \hat{h}_{a}(x)=\int \hat{p}_{t}(0, z) h_{a}(z) d z \leq a^{d} \int_{|z|<a \sqrt{d}}\left|\hat{p}_{t}(0, z)\right| d z .
$$

The function $p_{t}(0, z)$ has an explicit expression like (2.23). It follows from the expression that $\left|\hat{p}_{t}(0, z)\right| \leq\left|\hat{p}_{t}^{c}(0, z)\right|$ for any $c>0$. Hence transience of $\mathbf{X}$ follows if we show that

$$
\int_{0}^{\infty} d t \int_{|z|<1}\left|f_{t}^{c}(0, z)\right| d z<\infty
$$

for some $c>0$. Use $G(t, x)$ and $U(z)$ above. Then $\left|f_{t}^{c}(0, z)\right|=\exp G(t, x)$. Our task is to show that $\int_{|z|<1} U(z) d z<\infty$. Rewriting $U(z)$ as above and using Lemmas 2.1 and 2.4 again, we obtain

$$
\begin{gathered}
\int_{|z|<1} U(z) d z \leq \int_{|z|<1} d z \int_{0}^{1} \frac{d v}{v} \exp \left[\rho^{c}\left(\mathbf{R}^{d}\right)\left(\frac{K_{2}}{\alpha}+\frac{K_{5}}{\alpha} \log \frac{1}{\left|z_{1}\right|}+\frac{K_{6}}{\alpha}+K_{7}\right)\right. \\
\left.+\int \rho^{c}(d x) \int_{v}^{1}\left(e^{-u^{\alpha}|g(\log u ; x)|}-1\right) \frac{d u}{u}\right] \\
=C_{2} \int_{|z|<1}\left|z_{1}\right|^{-\alpha^{-1} K_{5} \rho^{c}\left(\mathbf{R}^{d}\right)} d z \int_{0}^{1} \frac{d v}{v} \exp \left[\int \rho^{c}(d x) \int_{v}^{1}\left(e^{-u^{\alpha}|g(\log u ; x)|}-1\right) \frac{d u}{u}\right]
\end{gathered}
$$

with some constant $C_{2}$. Now choose $c$ so large that $\alpha^{-1} K_{5} \rho^{c}\left(\mathbf{R}^{d}\right)<1$ and use (2.22). Then the last expression is finite. The proof is complete. 
Remark. Let $d=2$. The recurrence condition (1.5) reads as

$$
\int_{0}^{1} \frac{d v}{v} \exp \left[\int_{v}^{1} \frac{d u}{u} \int_{|x| \geq c}\left(e^{-u^{\alpha}\left|x_{1}+x_{2} \log u\right|}-1\right) \rho(d x)\right]=\infty .
$$

Let us compare it with the condition

$$
\int_{0}^{1} \frac{d v}{v} \exp \left[\int_{v}^{1} \frac{d u}{u} \int_{|x| \geq c}\left(e^{-u^{\alpha}\left(\left|x_{1}\right|+\left|x_{2} \log u\right|\right)}-1\right) \rho(d x)\right]=\infty .
$$

Clearly (2.25) implies (2.24). However, (2.24) does not imply (2.25). Indeed, we will prove in Section 4 the existence of a measure $\rho$ such that (2.24) is fulfilled but

$$
\int_{0}^{1} \frac{d v}{v} \exp \left[\int_{v}^{1} \frac{d u}{u} \int_{|x| \geq c}\left(e^{-u^{\alpha}|x|}-1\right) \rho(d x)\right]<\infty
$$

this $\rho$ satisfies, a fortiori, the condtion (2.26) with $e^{-u^{\alpha}|x|}$ replaced by $e^{-u^{\alpha}\left(\left|x_{1}\right|+\left|x_{2}\right|\right)}$, and so, does not satisfy (2.25).

\section{A unified form of recurrence criteria}

We prove Theorem B given in Section 1.

Proof of Theorem B. Our assumptions are that the eigenvalues of $Q$ are positive reals and that, with some invertible matrix $R$, one of the following two cases takes place:

Case 1. $R Q R^{-1}$ is diagonal.

Case 2. $R Q R^{-1}$ is a Jordan cell matrix.

Our assertion is that (1.6) is a recurrence criterion of $\mathbf{X}$.

Consider Case 1. Let $\alpha_{1}, \ldots, \alpha_{n}$ be the distinct eigenvalues of $Q$, and let $V_{j}$ be the eigenspace of $\alpha_{j}$. Let $I=T_{1}+\cdots+T_{n}$ be the decomposition of the identity matrix $I$ associated with the direct sum decomposition $\mathbf{R}^{d}=V_{1} \oplus \cdots \oplus V_{n}$. Thus $Q T, x=\alpha, T_{j} x$. Hence

$$
Q^{k} x=\sum_{j=1}^{n} \alpha_{j}^{k} T_{j} x \quad \text { and } \quad u^{Q} x=\sum_{j=1}^{n} u^{\alpha_{j}} T_{j} x .
$$

Define a norm $\|x\|$ by $\|x\|=\sum_{j=1}^{n}\left|T_{j} x\right|$. Then

$$
\left|u^{Q} x\right| \leq \sum_{j=1}^{n} u^{\alpha_{j}}\left|T_{j} x\right|=\left\|u^{Q} x\right\| .
$$


Sato, Watanabe, and Yamazato [1] prove that $\mathbf{X}$ is recurrent if and only if

$$
\int_{0}^{1} \frac{d v}{v} \exp \left[\int_{v}^{1} \frac{d u}{u} \int_{|x| \geq c}\left(e^{-\| u^{Q} x||}-1\right) \rho(d x)\right]=\infty .
$$

There is a constant $K>0$ such that $|x| \geq K\|x\|$ for every $x$. Define the condition (3.1)' by replacing $\left\|u^{Q} x\right\|$ in (3.1) by $K\left\|u^{Q} x\right\|$. Obviously, (3.1) implies (1.6) and (1.6) implies (3.1)'. Lemma 4.1 of [1] says that (3.1) and (3.1)' are equivalent. This settles Case 1 .

Next consider Case 2 . Let $G(s ; x)=\sum_{k=1}^{d}\left|g^{(k-1)}(s ; x)\right|$. First we remark that Theorem A remains valid if we replace $|g(\log u ; x)|$ in $(1.5)$ by $G(\log u ; x)$. To see this, we have only to repeat the proof of Theorem A using (2.16) in the place where we used (2.15). Let $R Q R^{-1}=S$. Since $(a R) Q(a R)^{-1}=S$ for every $a$ $>0$, we may assume that either $|R|$ or $\left|R^{-1}\right|$ equals a preassigned positive number (here the norm $|T|$ of a matrix $T$ is the norm as a linear operator). Since $u^{S}=R u^{Q} R^{-1}$, it follows from (1.1) that

$$
R \bar{X}_{t}=e^{-t S} R x+\int_{0}^{t} e^{-(t-r) s} d\left(R Z_{r}\right)
$$

Hence the process $R \mathbf{X}$ defined by $\left\{R \bar{X}_{t}\right\}$ is the OU type process associated with $\left\{R Z_{t}\right\}$ and $S$. The Lévy process $\left\{R Z_{t}\right\}$ has Lévy measure $\rho R^{-1}$ defined by $\left(\rho R^{-1}\right)(E)=\rho\{x: R x \in E\}$. The process $\mathbf{X}$ is recurrent if and only if $R \mathbf{X}$ is recurrent. So, the condition for recurrence is

$$
\int_{0}^{1} \frac{d v}{v} \exp \left[\int_{v}^{1} \frac{d u}{u} \int_{|x| \geq c}\left(e^{-u^{\alpha} G(\log u ; x)}-1\right)\left(\rho R^{-1}\right)(d x)\right]=\infty .
$$

Denote $\||x|\|=\sum_{j=1}^{d}\left|x_{j}\right|$ for $x=\left(x_{j}\right)_{1 \leq j \leq d}$. Then $u^{\alpha} G(\log u ; x)=\|\| u^{S} x \|$. If we assume that $\left|R^{-1}\right|=1$, then

$$
\begin{gathered}
\int_{|x| \geq c}\left(e^{-|| u^{s} x|| \mid}-1\right)\left(\rho R^{-1}\right)(d x) \leq \int_{|x| \geq c}\left(e^{-\left|u^{s} x\right|}-1\right)\left(\rho R^{-1}\right)(d x) \\
=\int_{|R x| \geq c}\left(e^{-\left|R u^{\ominus} x\right|}-1\right) \rho(d x) \leq \int_{|x| \geq c}\left(e^{-\left|u^{\natural} x\right|}-1\right) \rho(d x) .
\end{gathered}
$$

Hence (3.2) implies (1.6). If we assume that $|R|=1 / \sqrt{d}$, then

$$
\begin{gathered}
\int_{|x| \geq c}\left(e^{-|| u^{s} x|| \mid}-1\right)\left(\rho R^{-1}\right)(d x) \geq \int_{|x| \geq c}\left(e^{-\sqrt{d}\left|u^{s} x\right|}-1\right)\left(\rho R^{-1}\right)(d x) \\
=\int_{|R x| \geq c}\left(e^{-\sqrt{d}\left|R u^{0} x\right|}-1\right) \rho(d x) \geq \int_{|x| \geq \sqrt{d} c}\left(e^{-\left|u^{\natural} x\right|}-1\right) \rho(d x) .
\end{gathered}
$$


Let $\alpha$ be the eigenvalue of $Q$. For any $\varepsilon \in(0, \alpha)$ there is a constant $K$ such that

$$
\left|u^{Q} x\right| \leq K u^{\alpha-\varepsilon}|x| \text { for } 0<u \leq 1 \text { and } x \in \mathbf{R}^{d} .
$$

Therefore the condition (1.6) does not depend on the choice of $c$; this is shown by an argument similar to that given in the proof of Theorem A. It follows that (1.6) implies (3.2). The proof is complete.

\section{Comparison of the cases with diagonalizable and nondiagonalizable matrices in linear drift terms. Proof of Theorem C}

Let $d=2$ and let $Q_{0}$ and $Q_{1}$ be defined by (1.7). We compare OU type processes associated with $Q_{0}$ and $Q_{1}$ combined with a common Lévy preocess. First we prove (i) of Theorem C. Then, after two important lemmas, we establish (ii) of Theorem C.

Proof of (i) of Theorem C. We can choose a sequence $a_{1}=e<a_{2}<a_{3}<\cdots$ such that if we write $b_{n}=\log a_{n}$, then the following are satisfied:

$$
\begin{gathered}
\left(\frac{a_{2 n-1}}{a_{2 n}}\right)^{1 / b_{2 n-1}} b_{2 n} \leq e^{-n} \text { for } n \geq 1, \\
\left(\frac{b_{2 n-1}}{b_{2 n}}\right)^{1 / b_{2 n-1}} \leq e^{-n} \text { for } n \geq 1, \\
\left\{\prod_{j=1}^{n}\left(\frac{a_{2 j-1}}{a_{2 j}}\right)^{1 / b_{2 j-1}}\right\}\left\{\prod_{j=1}^{n-1} \frac{b_{2 j}}{b_{2 j+1}}\right\} b_{2 n} \log \frac{b_{2 n+1}}{b_{2 n}}=1 \text { for } n \geq 1,
\end{gathered}
$$

where, for $n=1, \Pi_{j=1}^{n-1}$ is understood to be 1 . Indeed, if $a_{1}, a_{2}, \ldots, a_{2 n-1}$ are found, we can find $a_{2 n}$ satisfying (4.1) and (4.2) and determine $a_{2 n+1}$ by (4.3). Clearly $a_{n}$ tends to infinity. Define $h(r)$ as follows:

$$
\begin{aligned}
& h(r)=1 / b_{2 n-1} \text { for } a_{2 n-1} \leq r<a_{2 n}, \\
& h(r)=1 / \log r \text { for } a_{2 n} \leq r<a_{2 n+1} .
\end{aligned}
$$

Then $h(r)$ decreases to 0 as $r \rightarrow \infty$. We claim that it satisfies (1.8) and (1.9). Let $c_{n}=1 / a_{n}$. If $c_{2 n}<v \leq c_{2 n-1}$, then

$$
\int_{e}^{1 / v} \frac{h(r)}{r} d r=\sum_{j=1}^{n-1} \frac{b_{2 j}-b_{2 j-1}}{b_{2 j-1}}+\sum_{j=1}^{n-1} \log \frac{b_{2 j+1}}{b_{2 j}}+\frac{\log (1 / v)}{b_{2 n-1}}-1 .
$$

If $c_{2 n+1}<v \leq c_{2 n}$, then 


$$
\begin{aligned}
\int_{e}^{1 / v} \frac{h(r)}{r} d r= & \sum_{j=1}^{n} \frac{b_{2 j}-b_{2 j-1}}{b_{2 j-1}}+\sum_{j=1}^{n-1} \log \frac{b_{2 j+1}}{b_{2 j}}+\log \frac{\log (1 / v)}{b_{2 n}}, \\
\int_{e}^{1 / v} \frac{h(r)}{r \log r} d r= & \sum_{j=1}^{n} \frac{1}{b_{2 j-1}} \log \frac{b_{2 j}}{b_{2 j-1}} \\
& +\sum_{j=1}^{n-1}\left(\frac{1}{b_{2 j}}-\frac{1}{b_{2 j+1}}\right)+\frac{1}{b_{2 n}}-\frac{1}{\log (1 / v)} .
\end{aligned}
$$

Let $n \geq 2$. It follows from (4.1) and (4.6) that

$$
\begin{aligned}
& \int_{c_{2 n}}^{c_{2 n-1}} \frac{d v}{v} \exp \left[-\int_{e}^{1 / v} \frac{h(r)}{r}\left(1+\frac{1}{\log r}\right) d r\right] \leq \int_{c_{2 n}}^{c_{2 n-1}} \frac{d v}{v} \exp \left[-\int_{e}^{1 / v} \frac{h(r)}{r} d r\right] \\
& =e\left\{\prod_{j=1}^{n-1}\left(\frac{a_{2 j-1}}{a_{2 j}}\right)^{1 / b_{2 j-1}}\right\}\left\{\prod_{j=1}^{n-2} \frac{b_{2 j}}{b_{2 j+1}}\right\} b_{2 n-2}\left(\left(\frac{1}{a_{2 n-1}}\right)^{1 / b_{2 n-1}}-\left(\frac{1}{a_{2 n}}\right)^{1 / b_{2 n-1}}\right) \\
& \leq e\left(\frac{a_{2 n-3}}{a_{2 n-2}}\right)^{1 / b_{2 n-3}} b_{2 n-2} \leq e^{2-n} .
\end{aligned}
$$

It follows from (4.7) that

$$
\int_{c_{2 n+1}}^{c_{2 n}} \frac{d v}{v} \exp \left[-\int_{e}^{1 / v} \frac{h(r)}{r} d r\right]=1,
$$

since the left-hand side of the above equals that of (4.3). Hence, using (4.2) and (4.8), we get

$$
\begin{gathered}
\int_{c_{2 n+1}}^{c_{2 n}} \frac{d v}{v} \exp \left[-\int_{e}^{1 / v} \frac{h(r)}{r}\left(1+\frac{1}{\log r}\right) d r\right] \leq \exp \left[-\int_{e}^{a_{2 n}} \frac{h(r)}{r \log r} d r\right] \\
=\left\{\prod_{j=1}^{n}\left(\frac{b_{2 j-1}}{b_{2 j}}\right)^{1 / b_{2 j-1}}\right\}\left\{\prod_{j=1}^{n-1} \exp \left(\frac{1}{b_{2 j+1}}-\frac{1}{b_{2 j}}\right)\right\} \leq\left(\frac{b_{2 n-1}}{b_{2 n}}\right)^{1 / b_{2 n-1}} \leq e^{-n}
\end{gathered}
$$

Therefore $h(r)$ satisfies (1.8) and (1.9).

Lemma 4.1 Suppose that $\rho$ is a finite measure on $\left\{x \in \mathbf{R}^{2}:\left|x_{2}\right|>\right.$ e $\}$. Let $\alpha>$ 0. Define $H(r), F_{0}(v)$, and $F_{1}(v)$ by

$$
\begin{gathered}
H(r)=\frac{1}{\alpha} \int_{\left|x_{2}\right|>r} \rho(d x), \\
F_{0}(v)=\int_{v}^{e^{-1}} \frac{d u}{u} \int_{\left|x_{2}\right|>e}\left(1-e^{-u^{\alpha}|x|}\right) \rho(d x), \\
F_{1}(v)=\int_{v}^{e^{-1}} \frac{d u}{u} \int_{\left|x_{2}\right|>e}\left(1-e^{-u^{\alpha}\left|x_{1}+x_{2} \log u\right|}\right) \rho(d x) .
\end{gathered}
$$


Assume that $\rho$ is concentrated on the set $E_{0}$ in Theorem $\mathrm{C}$. Then, as $v \downarrow 0$,

$$
\begin{gathered}
F_{0}(v)=\int_{e}^{v^{-\alpha}} \frac{H(r)}{r} d r+O(1), \\
F_{1}(v)=\int_{e}^{v^{-\alpha}|\log v|^{-1}} \frac{H(r)}{r}\left(1+\frac{1}{\log r}\right) d r+O(1) .
\end{gathered}
$$

Proof. Denote $c=x_{01} / x_{02}$ where $x_{01}$ and $x_{02}$ are given in Theorem C. Since $E_{0}=\left\{x=\left(\begin{array}{c}r c \\ r\end{array}\right):\left(\operatorname{sgn} x_{02}\right) r>0\right\}$, we have

$$
\begin{aligned}
& F_{0}(v)=\alpha \int_{v}^{e^{-1}} \frac{d u}{u} \int_{e}^{\infty}\left(e^{-r u^{\alpha}\left(1+c^{2}\right)^{1 / 2}}-1\right) d H(r), \\
& F_{1}(v)=\alpha \int_{v}^{e^{-1}} \frac{d u}{u} \int_{e}^{\infty}\left(e^{-r u^{\alpha}|c+\log u|}-1\right) d H(r) .
\end{aligned}
$$

Since

$$
\left|\int_{v}^{e^{-1}}\left(e^{-r u^{\alpha}\left(1+c^{2}\right)^{1 / 2}}-e^{-r u^{\alpha}}\right) \frac{d u}{u}\right| \leq \frac{1}{2 \alpha} \log \left(1+c^{2}\right)
$$

we have

$$
\begin{gathered}
F_{0}(v)=\alpha \int_{v}^{e^{-1}} \frac{d u}{u} \int_{e}^{\infty}\left(e^{-r u^{\alpha}}-1\right) d H(r)+O(1) \\
=\int_{v^{\alpha}}^{e^{-\alpha}} \frac{d u}{u} \int_{e}^{\infty}\left(e^{-r u}-1\right) d H(r)+O(1)=\int_{v^{\alpha}}^{e^{-\alpha}} d u \int_{e}^{\infty} e^{-r u} H(r) d r+O(1)
\end{gathered}
$$

by integration by parts. Below we will use the inequality $1-e^{-w} \leq w$ several times. We get

$$
\begin{aligned}
F_{0}(v) & =\int_{e}^{\infty} \frac{H(r)}{r} e^{-r v^{\alpha}} d r+O(1) \\
& =\int_{e}^{v^{-\alpha}} \frac{H(r)}{r} e^{-r v^{\alpha}} d r+O(1)=\int_{e}^{v^{-\alpha}} \frac{H(r)}{r} d r+O(1),
\end{aligned}
$$

which proves (4.12).

In order to show (4.13), denote by $F_{1}^{0}(v)$ the function $F_{1}(v)$ with $c=0$. Then $F_{1}^{0}(v)=\int_{v^{\alpha}}^{e^{-\alpha}} \frac{d u}{u} \int_{e}^{\infty}\left(e^{-r u|\log u| / \alpha}-1\right) d H(r)$ 


$$
\begin{gathered}
=\frac{1}{\alpha} \int_{v^{\alpha}}^{e^{-\alpha}}|\log u| d u \int_{e}^{\infty} e^{-r u|\log u| / \alpha} H(r) d r+O(1)=G_{0}(v)+G_{1}(v)+O(1), \\
G_{0}(v)=\frac{1}{\alpha} \int_{e}^{\infty} H(r) d r \int_{v^{\alpha}}^{e^{-\alpha}} e^{-r u|\log u| / \alpha}(|\log u|-1) d u, \\
G_{1}(v)=\frac{1}{\alpha} \int_{e}^{\infty} H(r) d r \int_{v^{\alpha}}^{e^{-\alpha}} e^{-r u|\log u| / \alpha} d u .
\end{gathered}
$$

We have

$$
G_{0}(v)=\int_{e}^{\infty} \frac{H(r)}{r} e^{-r v^{\alpha}|\log v|} d r+\text { const }=\int_{e}^{v^{-\alpha}|\log v|^{-1}} \frac{H(r)}{r} d r+O(1) .
$$

We claim that

$$
G_{1}(v)=\int_{e}^{v^{-\alpha}|\log v|^{-1}} \frac{H(r)}{r \log r} d r+O(1)
$$

Make substitution $u|\log u|=w$ for $0<u \leq e^{-e}$. Note that $\frac{d w}{d u}=|\log u|-1 \geq$ $e-1$. Let $a=e^{1-e} \wedge \alpha e^{-1}$. Then

$$
\begin{aligned}
G_{1}(v) & =\frac{1}{\alpha} \int_{\alpha v^{\alpha}|\log v|}^{a} \frac{d w}{|\log u|-1} \int_{e}^{\infty} H(r) e^{-r w / \alpha} d r+\text { const } \\
& =\frac{1}{\alpha} \int_{\alpha v^{\alpha}|\log v|}^{a} \frac{d w}{|\log w|} \int_{e}^{\infty} H(r) e^{-r w / \alpha} d r+O(1) .
\end{aligned}
$$

Here we employed that

$$
\begin{gathered}
\left|\frac{1}{|\log u|-1}-\frac{1}{|\log w|}\right|=\frac{\log |\log u|-1}{(|\log u|-1)|\log w|} \\
\log |\log u|-1 \leq \log |\log w|, \quad|\log u|-1 \geq|\log w| / 2, \\
\int_{0}^{a} \frac{\log |\log w|}{(\log w)^{2}} d w \int_{e}^{\infty} H(r) e^{-r w / \alpha} d r \leq \alpha H(e) \int_{0}^{a} \frac{\log |\log w|}{w(\log w)^{2}} d w<\infty .
\end{gathered}
$$

Next, using integration by parts, we get

$$
\begin{aligned}
G_{1}(v)= & \int_{e}^{\infty} \frac{H(r)}{r} \frac{e^{-r v^{\alpha}|\log v|}}{\left|\log \left(\alpha v^{\alpha}|\log v|\right)\right|} d r \\
& +\int_{e}^{\infty} \frac{H(r)}{r} d r \int_{\alpha v^{\alpha}|\log v|}^{a} \frac{e^{-r w / \alpha}}{w(\log w)^{2}} d w+O(1) .
\end{aligned}
$$

The first term of the right-hand side is bounded, as is seen by splitting the inte- 
gral at $v^{-\alpha}|\log v|^{-1}$. Since

$$
\begin{gathered}
\int_{\alpha v^{\alpha}|\log v|}^{a} \frac{d w}{w(\log w)^{2}} \int_{e}^{a / w} \frac{H(r)}{r}\left(1-e^{-r w / \alpha}\right) d r \leq \frac{1}{\alpha} \int_{0}^{a} \frac{d w}{(\log w)^{2}} \int_{e}^{\alpha / w} H(r) d r, \\
\int_{\alpha v^{\alpha}|\log v|}^{a} \frac{d w}{w(\log w)^{2}} \int_{\alpha / w}^{\infty} \frac{H(r)}{r} e^{-r w / \alpha} d r \leq H(e) \int_{0}^{a} \frac{d w}{w(\log w)^{2}} \int_{1}^{\infty} \frac{e^{-s}}{s} d s,
\end{gathered}
$$

we get

$$
\begin{aligned}
G_{1}(v) & =\int_{\alpha v^{\alpha}|\log v|}^{a} \frac{d w}{w(\log w)^{2}} \int_{e}^{a / w} \frac{H(r)}{r} d r+O(1) \\
& =\int_{\alpha / a}^{v^{-\alpha}|\log v|^{-1}} \frac{d w}{w(\log (w / \alpha))^{2}} \int_{e}^{w} \frac{H(r)}{r}+O(1)
\end{aligned}
$$

By integration by parts again,

$$
G_{1}(v)=\int_{\alpha / a}^{v^{-\alpha}|\log v|^{-1}} \frac{H(w)}{w \log (w / \alpha)} d w+O(1)
$$

which gives (4.16) since

$$
\left|\frac{1}{\log (w / \alpha)}-\frac{1}{\log w}\right| \leq \frac{\text { const }}{(\log w)^{2}}
$$

Therefore (4.13) is obtained for $F_{1}^{0}(v)$. Now consider the case $c \neq 0$. Let $b=e^{c}$. Then, from (4.15),

$$
F_{1}(v)=\alpha \int_{b v}^{b / e} \frac{d u}{u} \int_{e}^{\infty}\left(e^{-r(u / b)^{\alpha}|\log u|}-1\right) d H(r) .
$$

Since

$$
\left|\int_{b v}^{b / e}\left(e^{-r(u / b)^{\alpha}|\log u|}-e^{-r u^{\alpha}|\log u|}\right) \frac{d u}{u}\right| \leq \frac{1}{\alpha}\left(K_{3} \alpha|c|+K_{4}\right)
$$

by Lemma 2.2 , we get

$$
\begin{aligned}
F_{1}(v) & =\alpha \int_{b v}^{b / e} \frac{d u}{u} \int_{e}^{\infty}\left(e^{-r u^{\alpha}|\log u|}-1\right) d H(r)+O(1) \\
& =\alpha \int_{v}^{1 / e} \frac{d u}{u} \int_{e}^{\infty}\left(e^{-r u^{\alpha}|\log u|}-1\right) d H(r)+O(1)=F_{1}^{0}(v)+O(1),
\end{aligned}
$$

using $\int_{b v}^{v} \frac{d u}{u}=c$. Hence (4.13). 
Lemma 4.2. Let $H(r), F_{0}(v), F_{1}(v)$ be defined by (4.9)-(4.11). Assume that $\rho$ is concentrated on the set $E_{1}$ in Theorem $\mathrm{C}$. Then, as $v \downarrow 0$,

$$
\begin{gathered}
F_{0}(v)=\int_{e}^{v^{-\alpha}|\log v|^{-1}} \frac{H(r)}{r}\left(1+\frac{1}{\log r}\right) d r+O(1), \\
F_{1}(v)=\int_{e}^{v^{-\alpha}} \frac{H(r)}{r} d r+O(1) .
\end{gathered}
$$

Proof. Denote $c=\frac{x_{01}}{x_{02}}-\alpha^{-1} \log \left|x_{02}\right|$. Then

$$
E_{1}=\left\{x=\left(\begin{array}{c}
c r+\alpha^{-1} r \log |r| \\
r
\end{array}\right):\left(\operatorname{sgn} x_{02}\right) r>0\right\} .
$$

Hence

$$
\begin{aligned}
& F_{0}(v)=\alpha \int_{v}^{e^{-1}} \frac{d u}{u} \int_{e}^{\infty}\left(e^{-u^{\alpha} r\left(1+\left(c+\alpha^{-1} \log r\right)^{2}\right)^{1 / 2}}-1\right) d H(r), \\
& F_{1}(v)=\alpha \int_{v}^{e^{-1}} \frac{d u}{u} \int_{e}^{\infty}\left(e^{-u^{\alpha} r\left|c+\log \left(u r^{1 / \alpha}\right)\right|}-1\right) d H(r)
\end{aligned}
$$

Let us show (4.17). We have

$$
\begin{gathered}
\left|\int_{v}^{e^{-1}}\left(e^{-u^{\alpha} r\left(1+\left(c+\alpha^{-1} \log r\right)^{2}\right)^{1 / 2}}-e^{-\alpha^{-1} u^{\alpha} r \log r}\right) \frac{d u}{u}\right| \\
\leq \frac{1}{\alpha}\left|\log \frac{\left(1+\left(c+\alpha^{-1} \log r\right)^{2}\right)^{1 / 2}}{\alpha^{-1} \log r}\right| \leq K
\end{gathered}
$$

for $r>e$ with a constant $K$ independent of $v$ and $r$. Therefore

$$
\begin{aligned}
F_{0}(v) & =\alpha \int_{v}^{e^{-1}} \frac{d u}{u} \int_{e}^{\infty}\left(e^{-\alpha^{-1} u^{\alpha} r \log r}-1\right) d H(r)+O(1) \\
& =\int_{v}^{e^{-1}} u^{\alpha-1} d u \int_{e}^{\infty} H(r)(1+\log r) e^{-\alpha^{-1} u^{\alpha} r \log r} d r+O(1) \\
& =\int_{e}^{\infty} \frac{H(r)}{r}\left(1+\frac{1}{\log r}\right) e^{-\alpha^{-1} v^{\alpha} r \log r} d r+O(1)
\end{aligned}
$$

Let $s=\alpha^{-1} v^{\alpha} r \log r$ and $s_{v}=\alpha^{-1}|\log v|^{-1} \log \left(v^{-\alpha}|\log v|^{-1}\right)$. We have $s^{-1} d s=$ $\left(1+(\log r)^{-1}\right) r^{-1} d r$, and

$$
\int_{v^{-\alpha}|\log v|^{-1}}^{\infty} \frac{H(r)}{r}\left(1+\frac{1}{\log r}\right) e^{-\alpha^{-1} v^{\alpha_{r} \log r}} d r=\int_{s_{v}}^{\infty} H(r) e^{-s} \frac{d s}{s}=O(1)
$$




$$
\begin{aligned}
\int_{e}^{v^{-\alpha}|\log v|^{-1}} \frac{H(r)}{r} & \left(1+\frac{1}{\log r}\right)\left(1-e^{-\alpha^{-1} v^{\alpha} r \log r}\right) d r \\
= & \int_{e \alpha^{-1} v^{\alpha}}^{s_{v}} H(r)\left(1-e^{-s}\right) \frac{d s}{s}=O(1),
\end{aligned}
$$

since $s_{v} \rightarrow 1$ as $v \downarrow 0$. Hence we get (4.17).

In order to prove (4.18) in case $c=0$, denote by $F_{1}^{0}(v)$ the function $F_{1}(v)$ with $c=0$. Write

$$
F_{1}^{0}(v)=G_{0}(v)+G_{1}(v)+\text { const, }
$$

where we define, with $a=e^{-1 / \alpha}$,

$$
\begin{aligned}
& G_{0}(v)=\alpha \int_{v}^{a} \frac{d u}{u} \int_{e}^{u^{-\alpha}}\left(e^{u^{\alpha} r \log \left(u r^{1 / \alpha}\right)}-1\right) d H(r), \\
& G_{1}(v)=\alpha \int_{v}^{a} \frac{d u}{u} \int_{u^{-\alpha}}^{\infty}\left(e^{-u^{\alpha} r \log \left(u r^{1 / \alpha}\right)}-1\right) d H(r) .
\end{aligned}
$$

We have

$$
\begin{aligned}
G_{0}(v) & =\alpha \int_{v}^{a} u^{\alpha-1} d u \int_{e}^{u^{-\alpha}} H(r)\left(-\log \left(u r^{1 / \alpha}\right)-\frac{1}{\alpha}\right) e^{u^{\alpha_{r} \log \left(u r^{1 / \alpha}\right)}} d r+O(1) \\
& =\int_{e}^{v^{-\alpha}} \frac{H(r)}{r}\left(e^{v^{\alpha_{r} \log \left(v r^{1 / \alpha}\right)}}-1\right) d r+O(1)
\end{aligned}
$$

and hence

$$
\begin{aligned}
\left|G_{0}(v)\right| & \leq \alpha H(e) \int_{v / a}^{1}\left(1-e^{s^{\alpha} \log s}\right) \frac{d s}{s}+O(1) \\
& \leq \alpha H(e) \int_{0}^{1} s^{\alpha-1}|\log s| d s+O(1)=O(1) .
\end{aligned}
$$

On the other hand,

$$
\begin{aligned}
G_{1}(v)= & \alpha \int_{v}^{a} u^{\alpha-1} d u \int_{u^{-\alpha}}^{\infty} H(r)\left(\log \left(u r^{1 / \alpha}\right)+\frac{1}{\alpha}\right) e^{-u^{\alpha} r \log \left(u r^{1 / \alpha}\right)} d r \\
= & \int_{e}^{v^{-\alpha}} \frac{H(r)}{r}\left(1-e^{-(\alpha e)^{-1} r \log \left(e^{-1} r\right)}\right) d r \\
& +\int_{v^{-\alpha}}^{\infty} \frac{H(r)}{r}\left(e^{-v^{\alpha} r \log \left(v r^{1 / \alpha}\right)}-e^{-(\alpha e)^{-1} r \log \left(e^{-1} r\right)}\right) d r \\
= & \int_{e}^{v^{-\alpha}} \frac{H(r)}{r} d r+\int_{v^{-\alpha}}^{\infty} \frac{H(r)}{r} e^{-v^{\alpha} r \log \left(v r^{1 / \alpha}\right)} d r+O(1) .
\end{aligned}
$$


The second term of the last line is bounded, as is seen from substitution $v r^{1 / \alpha}=$ $s$. Hence we obtain (4.18) for $F_{1}^{0}(v)$. To handle $F_{1}(v)$ in case $c \neq 0$, let $b=e^{c}$. Then

$$
\begin{aligned}
F_{1}(v) & =\int_{(b v)^{\alpha}}^{(b / e)^{\alpha}} \frac{d u}{u} \int_{e}^{\infty}\left(e^{-\alpha^{-1} b^{-\alpha} u r|\log u r|}-1\right) d H(r) \\
& =\int_{(b v)^{\alpha}}^{(b / e)^{\alpha}} \frac{d u}{u} \int_{e}^{\infty}\left(e^{-\alpha^{-1} u r \mid \log u r^{\prime}}-1\right) d H(r)+O(1)
\end{aligned}
$$

since

$$
\left|\int_{(b v)^{\alpha}}^{(b / e)^{\alpha}}\left(e^{-\alpha^{-1} b^{-\alpha} u r|\log u r|}-e^{-\alpha^{-1} u r|\log u r|}\right) \frac{d u}{u}\right| \leq K_{3} \alpha|c|+K_{4}
$$

by Lemma 2.2 . Hence

$$
F_{1}(v)=\int_{v^{\alpha}}^{e^{-\alpha}} \frac{d u}{u} \int_{e}^{\infty}\left(e^{-\alpha^{-1} u r|\log u r|}-1\right) d H(r)+O(1)=F_{1}^{0}(v)+O(1),
$$

completing the proof.

Proof of (ii) of Theorem C. We assume that the restriction $[\rho]_{\{|x|>c\}}$ of the Lévy measure $\rho$ is concentrated on $E_{0}$ or $E_{1}$. It follows from the criteria in Theorems $\mathrm{A}$ and $\mathrm{B}$ that, for any bounded set $E$, the measure $[\rho]_{E}$ is irrelevant to recurrence and transience. Hence we may and do assume that $\rho$ itself is concentrated on $E_{0}$ or $E_{1}$ and that $[\rho]_{\left\{\left|x_{2}\right| \leq e\right\}}=0$. Denote

$$
\begin{gathered}
I_{0}=\int_{0}^{a} \frac{d v}{v} \exp \left[\int_{v}^{a} \frac{d u}{u} \int_{\left|x_{2}\right|>e}\left(e^{-u^{\alpha}|x|}-1\right) \rho(d x)\right], \\
I_{1}=\int_{0}^{a} \frac{d v}{v} \exp \left[\int_{v}^{a} \frac{d u}{u} \int_{\left|x_{2}\right|>e}\left(e^{-u^{\alpha}\left|x_{1}+x_{2} \log u\right|}-1\right) \rho(d x)\right],
\end{gathered}
$$

where $a$ is an arbitrarily fixed positive real. The recurrence conditions of $\mathbf{X}_{0}$ and $\mathbf{X}_{1}$ are, respectively, $I_{0}=\infty$ and $I_{1}=\infty$. Define $H(r)$ by (4.9) and denote

$$
\begin{aligned}
& J_{0}=\int_{0}^{e^{-1}} \frac{d v}{v} \exp \left[-\int_{e}^{v^{-1}} \frac{H(r)}{r} d r\right] \\
& J_{1}=\int_{0}^{e^{-1}} \frac{d v}{v} \exp \left[-\int_{e}^{v^{-1}} \frac{H(r)}{r}\left(1+\frac{1}{\log r}\right) d r\right] .
\end{aligned}
$$

To prove the first assertion in (ii), assume that $\rho$ is concentrated on $E_{0}$. Then we claim that 


$$
\begin{aligned}
& I_{0}=\infty \text { if and only if } J_{0}=\infty, \\
& I_{1}=\infty \text { if and only if } J_{1}=\infty .
\end{aligned}
$$

It follows from (4.12) of Lemma 4.1 that $I_{0}=\infty$ is equivalent to

$$
\int_{0}^{a} \frac{d v}{v} \exp \left[-\int_{e}^{v^{-\alpha}} \frac{H(r)}{r} d r\right]=\infty .
$$

Hence (4.21) follows. Likewise, using (4.13) of Lemma 4.1, we see that $I_{1}=\infty$ is equivalent to

$$
\int_{0}^{a} \frac{d v}{v} \exp \left[-\int_{e}^{v^{-\alpha}|\log v|^{-1}} \frac{H(r)}{r}\left(1+\frac{r}{\log r}\right) d r\right]=\infty .
$$

Choose $a$ so small that $v^{\alpha}|\log v|$ is strictly increasing on (0, $a$ ]. Let $v^{\alpha}|\log v|$ $=w$. Since $(\alpha|\log v|-1)|\log v|^{-1} v^{-1} d v=w^{-1} d w,(4.23)$ is equivalent to $J_{1}=$ $\infty$. This shows (4.22). Now, if (1.8) and (1.9) are satisfied with $H(r)$ in place of $h(r)$, then $\mathbf{X}_{0}$ is recurrent and $\mathbf{X}_{1}$ is transient.

To prove the second assertion in (ii), we assume that $\rho$ is concentrated on $E_{1}$. Then, using Lemma 4.2 instead of Lemma 4.1, the same argument shows that

$$
\begin{aligned}
& I_{0}=\infty \text { if and only if } J_{1}=\infty, \\
& I_{1}=\infty \text { if and only if } J_{0}=\infty .
\end{aligned}
$$

If we have (1.8) and (1.9) with $H(r)$ in place of $h(r)$, then (4.24) and (4.25) show that $\mathbf{X}_{0}$ is transient and $\mathbf{X}_{1}$ is recurrent. The proof is complete.

\section{Comparison of the cases with diagonalizable and nondiagonalizable matrices in linear drift terms. Proof of Theorem D}

We continue to assume that $d=2, Q_{0}$ and $Q_{1}$ are given by (1.7), and $\mathbf{X}_{0}$ and $\mathbf{X}_{1}$ are the OU type processes associated with $Q_{0}$ and $Q_{1}$, respectively, combined with a common Lévy process $\left\{Z_{t}\right\}$. We will prove Theorem D, using Lemma 5.2. The proof of Lemma 5.2 needs Lemma 5.1. Delicate estimate of integrals is required.

In the following two lemmas let $M$ be a positive real and $H(r)$ be a function which is defined on $[e, \infty)$, nonnegative, right-continuous, and decreasing, and which satisfies

$$
H(e)+\int_{e}^{\infty} \frac{H(r)}{r \log r} d r \leq M
$$


that is,

$$
-\int_{e}^{\infty}(1+\log \log r) d H(r) \leq M
$$

It follows that

$$
H(r) \leq \frac{1}{\log \log r} \int_{e}^{r} \frac{H(s)}{s \log s} d s \leq \frac{M}{\log \log r} \text { for } r>e .
$$

Let us denote by $K_{1}, K_{2}, \ldots$ constants that depend only on $M$.

Lemma 5.1. Let $0<\varepsilon<1 / 2$. If $0<v<e^{-2}$ and $|1+\varepsilon \log v| \geq \varepsilon$, then

$$
\left|\int_{v^{-1}}^{v^{-1}|1+\varepsilon \log v|^{-1}} \frac{H(r)}{r} d r\right| \leq K_{1}
$$

Proof. Denote by $J$ the integral in the left-hand side of (5.4). Cosider three cases.

Case 1: $\varepsilon \leq|1+\varepsilon \log v| \leq 1 / 2$.

Case 2: $1 / 2<|1+\varepsilon \log v| \leq 1$.

Case 3: $|1+\varepsilon \log v|>1$.

In Case 1 , note that $(1 / 2)|\log v|^{-1} \leq \varepsilon \leq(3 / 2)|\log v|^{-1}$ and obtain

$$
|J| \leq H\left(v^{-1}\right) \int_{v^{-1}}^{(\varepsilon v)^{-1}} \frac{d r}{r} \leq \frac{M|\log \varepsilon|}{\log |\log v|} \leq \frac{M \log |2 \log v|}{\log |\log v|} \leq K_{2},
$$

using (5.3). In Case 2 we get

$$
|J| \leq H(e) \int_{v^{-1}}^{2 v^{-1}} \frac{d r}{r} \leq M \log 2=K_{3} .
$$

In Case 3, note that $|1+\varepsilon \log v|=\varepsilon|\log v|-1<|\log v|$, and

$$
|J| \leq \int_{v^{-1}|\log v|^{-1}}^{v^{-1}} \frac{H(r)}{r} d r \leq H\left(v^{-1}|\log v|^{-1}\right) \log |\log v| \leq \frac{M \log |\log v|}{\log \log |v \log v|} \leq K_{4} .
$$

These together prove (5.4).

Lemma 5.2. Let $c>0$ and $0 \leq \varepsilon<1 / 2$. Define, for $v \in\left(0, e^{-1}\right)$,

$$
F(v)=\int_{v}^{e^{-1}} \frac{d u}{u} \int_{e}^{\infty}\left(e^{-c r u|1+\varepsilon \log u|}-1\right) d H(r)
$$


Then, for all $v$ and $\varepsilon$,

$$
\left|F(v)-\int_{e}^{v^{-1}} \frac{H(r)}{r} d r\right| \leq K_{5}+K_{6}|\log c|
$$

Proof. Since $F(v)$ is continuous in $\varepsilon$, we may and do assume that $0<\varepsilon<1 / 2$. Denote $F(v)$ with $c=1$ by $F^{1}(v)$. First notice that

$$
\begin{aligned}
\mid F(v) & -F^{1}(v)|=| \int_{v}^{e^{-1}} \frac{d u}{u} \int_{e}^{\infty}\left(e^{-c r u|1+\varepsilon \log u|}-e^{-r u|1+\varepsilon \log u|}\right) d H(r) \mid \\
& \leq-\int_{e}^{\infty} d H(r)\left|\int_{0}^{e^{-1}}\left(e^{-c r u|1+\varepsilon \log u|}-e^{-r u|1+\varepsilon \log u|}\right) \frac{d u}{u}\right| \\
& \leq H(e)\left(C_{1}|\log c|+C_{2}\right)
\end{aligned}
$$

with absolute constants $C_{1}$ and $C_{2}$ by Lemma 2.2. Thus it is enough to consider only $F^{1}$. Notice also that we may assume that $v \in\left(0, e^{-2}\right)$, because (5.6) is evident if $v \in\left[e^{-2}, e^{-1}\right)$. Let $a(\varepsilon)=e^{-1 / \varepsilon}$. We split the proof into three cases.

Case 1: $e^{-1}>v \geq a(\varepsilon) e$.

Case 2: $a(\varepsilon) e>v \geq a(\varepsilon) e^{-1}$.

Case 3: $a(\varepsilon) e^{-1}>v>0$.

Case 1 . We have $1+\varepsilon \log v \geq \varepsilon$. Use integration by parts. We get $F^{1}(v)=$ $G_{1}+G_{2}-G_{3}$ with

$$
\begin{aligned}
G_{1} & =\int_{v}^{e^{-1}}\left(1-e^{-e u(1+\varepsilon \log u)}\right) H(e) \frac{d u}{u}, \\
G_{2} & =\int_{v}^{e^{-1}}(1+\varepsilon \log u+\varepsilon) d u \int_{e}^{\infty} e^{-r u(1+\varepsilon \log u)} H(r) d r, \\
G_{3} & =\varepsilon \int_{v}^{e^{-1}} d u \int_{e}^{\infty} e^{-r u(1+\varepsilon \log u)} H(r) d r .
\end{aligned}
$$

First,

$$
0 \leq G_{1} \leq \int_{v}^{e^{-1}}(1+\varepsilon \log u) e H(e) d u \leq K_{7}
$$

Secondly, by Lemma 5.1,

$$
\left|G_{2}-\int_{e}^{v^{-1}} \frac{H(r)}{r} d r\right| \leq K_{8},
$$

since 


$$
\begin{aligned}
G_{2}= & \int_{e}^{\infty} \frac{H(r)}{r}\left(e^{-r v(1+\varepsilon \log v)}-e^{-(1-\varepsilon) r / e}\right) d r=\int_{e}^{v^{-1}(1+\varepsilon \log v)^{-1}} \frac{H(r)}{r} d r+R, \\
|R| \leq & \int_{e}^{\infty} \frac{H(r)}{r} e^{-(1-\varepsilon) r / e} d r+\int_{v^{-1}(1+\varepsilon \log v)^{-1}}^{\infty} \frac{H(r)}{r} e^{-r v(1+\varepsilon \log v)} d r \\
& +\int_{e}^{v^{-1}(1+\varepsilon \log v)^{-1}} \frac{H(r)}{r}\left(1-e^{-r v(1+\varepsilon \log v)}\right) d r \leq K_{9} .
\end{aligned}
$$

Thirdly, let us handle $G_{3}$. Let $y=u / a(\varepsilon)$. Write $\varepsilon a(\varepsilon)=A$ for brevity. Since $G_{3}$ is greatest when $v=a(\varepsilon) e$,

$$
0 \leq G_{3} \leq A \int_{e}^{1 /(a(\varepsilon) e)} d y \int_{e}^{\infty} e^{-A r y \log y} H(r) d r .
$$

Let $w=y \log y$ and note that $\frac{d w}{d y}=\log y+1 \geq \frac{1}{2} \log w$. Then

$$
G_{3} \leq 2 A \int_{e}^{\infty} \frac{d w}{\log w} \int_{e}^{\infty} e^{-A w r} H(r) d r=U_{1}+U_{2},
$$

where

$$
U_{1}=2 A \int_{e}^{\infty} \frac{d w}{\log w} \int_{e}^{w} e^{-A w r} H(r) d r, \quad U_{2}=2 A \int_{e}^{\infty} \frac{d w}{\log w} \int_{w}^{\infty} e^{-A w r} H(r) d r .
$$

Now

$$
\begin{aligned}
U_{1} & =2 A \int_{e}^{\infty} H(r) d r \int_{r}^{\infty} \frac{e^{-A w r}}{\log w} d w \\
& =2 A \int_{e}^{\infty} H(r) d r\left\{\left[\frac{-e^{-A w r}}{A r \log w}\right]_{r}^{\infty}-\int_{r}^{\infty} \frac{e^{-A w r}}{A r w(\log w)^{2}} d w\right\} \\
& \leq 2 \int_{e}^{\infty} \frac{H(r)}{r \log r} e^{-A r^{2}} d r \leq 2 M, \\
U_{2} & \leq 2 A \int_{e}^{\infty} \frac{H(w)}{\log w} d w \int_{w}^{\infty} e^{-A w r} d r=2 \int_{e}^{\infty} \frac{H(w)}{w \log w} e^{-A w^{2}} d w \leq 2 M .
\end{aligned}
$$

Hence $0 \leq G_{3} \leq 4 M$. Combined with (5.7) and (5.8), this shows (5.6) in Case 1 .

Case 2. Since $a(\varepsilon) e>v \geq \alpha(\varepsilon) / e$, we have

$$
0 \leq F^{1}(v)-F^{1}(a(\varepsilon) e) \leq H(e) \int_{a(\varepsilon) / e}^{a(\varepsilon) e} \frac{d u}{u} \leq 2 M, \quad \int_{1 /(a(\varepsilon) e)}^{v^{-1}} \frac{H(r)}{r} d r \leq 2 M .
$$

Therefore (5.6) in this case follows from (5.6) for $v=a(\varepsilon) e$.

Case 3. In this case we have $1+\varepsilon \log v<-\varepsilon$ and 


$$
\begin{aligned}
F^{1}(v)= & F^{1}(a(\varepsilon) / e)+\int_{v}^{a(\varepsilon) / e} \frac{d u}{u} \int_{e}^{\infty}\left(e^{-r u \mid 1+\varepsilon \log u\}}-1\right) d H(r) \\
= & F^{1}(a(\varepsilon) / e)+\int_{v}^{a(\varepsilon) / e} \frac{d u}{u}\left\{\left[\left(e^{-r u|1+\varepsilon \log u|}-1\right) H(r)\right]_{e}^{\infty}\right. \\
& \left.+u|1+\varepsilon \log u| \int_{e}^{\infty} e^{-r u|1+\varepsilon \log u|} H(r) d r\right\} .
\end{aligned}
$$

Hence

$$
\begin{aligned}
F^{1}(v) & =F^{1}(a(\varepsilon) / e)+G_{1}+G_{2}+G_{3}, \\
G_{1} & =H(e) \int_{v}^{a(\varepsilon) / e}\left(1-e^{-e u|1+\varepsilon \log u|}\right) \frac{d u}{u}, \\
G_{2} & =\int_{v}^{a(\varepsilon) / e}(-\varepsilon \log u-1-\varepsilon) d u \int_{e}^{\infty} e^{-r u|1+\varepsilon \log u|} H(r) d r, \\
G_{3} & =\varepsilon \int_{v}^{a(\varepsilon) / e} d u \int_{e}^{\infty} e^{-r u|1+\varepsilon \log u|} H(r) d r .
\end{aligned}
$$

It follows from Case 2 that

$$
\left|F^{1}(a(\varepsilon) / e)-\int_{e}^{e / a(\varepsilon)} \frac{H(r)}{r} d r\right| \leq K_{10} .
$$

Obviously

$$
0 \leq G_{1} \leq H(e) \int_{v}^{a(\varepsilon) / e} e|1+\varepsilon \log u| d u \leq K_{11} .
$$

Concerning $G_{2}$ and $G_{3}$ let us show that

$$
\begin{gathered}
\left|G_{2}-\int_{e / a(\varepsilon)}^{v^{-1}} \frac{H(r)}{r} d r\right| \leq K_{12}, \\
0 \leq G_{3} \leq K_{13} .
\end{gathered}
$$

We have

$$
\begin{aligned}
G_{2} & =\int_{e}^{\infty} \frac{H(r)}{r} e^{-r v|1+\varepsilon \log v|} d r-\int_{e}^{\infty} \frac{H(r)}{r} e^{-r a(\varepsilon) \varepsilon / e} d r \\
& =\int_{e}^{v^{-1}|1+\varepsilon \log v|^{-1}} \frac{H(r)}{r} d r-\int_{e}^{e /(a(\varepsilon) \varepsilon)} \frac{H(r)}{r} d r+R_{1} \\
& =\int_{e}^{v^{-1}} \frac{H(r)}{r} d r-\int_{e}^{e / a(\varepsilon)} \frac{H(r)}{r} d r+R_{2}
\end{aligned}
$$


with $\left|R_{1}\right|$ and $\left|R_{2}\right|$ bounded by some $K_{12}$. Here we applied Lemma 5.1 twice: to $v$ directly and to $a(\varepsilon) e^{-1}$, noting that $a(\varepsilon) \varepsilon e^{-1}=a(\varepsilon) e^{-1}\left|1+\varepsilon \log \left(a(\varepsilon) e^{-1}\right)\right|$. Thus (5.11) was proved. Write $A=a(\varepsilon) \varepsilon$. Let $y=u / a(\varepsilon)$. Then

$$
\begin{aligned}
0 \leq G_{3} & \leq A \int_{0}^{e^{-1}} d y \int_{e}^{\infty} e^{-A r y|\log y|} H(r) d r=U_{1}+U_{2}, \\
U_{1} & =A \int_{e^{-2}}^{e^{-1}} d y \int_{e}^{\infty} e^{-A r y|\log y|} H(r) d r \\
U_{2} & =A \int_{0}^{e^{-2}} d y \int_{e}^{\infty} e^{-A r y|\log y|} H(r) d r .
\end{aligned}
$$

Now

$$
U_{1} \leq H(e) \int_{e^{-2}}^{e^{-1}} \frac{d y}{y|\log y|} \leq K_{14}
$$

Let $y|\log y|=w$ for $0<y<e^{-2}$. Then $\frac{d w}{d y}=|\log y|-1 \geq|\log w| / 2$. Hence

$$
U_{2} \leq 2 A \int_{0}^{2 e^{-2}} \frac{d w}{\lceil\log w\rceil} \int_{e}^{\infty} e^{-A w r} H(r) d r \leq V_{1}+V_{2}
$$

where

$$
\begin{aligned}
& V_{1}=2 A \int_{0}^{e^{-1}} \frac{d w}{\log w\rceil} \int_{e}^{w^{-1}} e^{-A w r} H(r) d r \\
& V_{2}=2 A \int_{0}^{e^{-1}} \frac{d w}{\log w} \int_{w^{-1}}^{\infty} e^{-A w r} H(r) d r
\end{aligned}
$$

Notice that

$$
\begin{aligned}
& V_{1} \leq 2 A \int_{0}^{e^{-1}} \frac{d w}{\lceil\log w\rceil} \int_{e}^{w^{-1}} H(r) d r=2 A \int_{0}^{e^{-1}} \frac{H\left(w^{-1}\right)}{w^{2}} d w \int_{0}^{w} \frac{d u}{\lceil\log u} \\
& \leq 2 A \int_{0}^{e^{-1}} \frac{H\left(w^{-1}\right)}{w|\log w|} d w=2 A \int_{e}^{\infty} \frac{H(r)}{r \log r} d r \leq 2 A M, \\
& V_{2} \leq 2 A \int_{0}^{e^{-1}} \frac{H\left(w^{-1}\right)}{\mid \log w\rceil} d w \int_{w^{-1}}^{\infty} e^{-A w r} d r=2 e^{-A} \int_{0}^{e^{-1}} \frac{H\left(w^{-1}\right)}{w|\log w|} d w \leq 2 e^{-A} M .
\end{aligned}
$$

Then (5.12) follows. Now (5.9)-(5.12) together imply (5.6) in Case 3.

Proof of Theorem D. We assume (1.11) for the Lévy measure $\rho$ of $\left\{Z_{t}\right\}$. Define $I_{0}$ and $I_{1}$ by (4.19) and (4.20) replacing the domain of integration $\left\{\left|x_{2}\right|>e\right\}$ 
with $\{|x|>e\}$ and letting $a=e^{-1}$. Then, by Theorems A and $\mathrm{B}, I_{0}=\infty$ and $I_{1}$ $=\infty$ are recurrence conditions of $\mathbf{X}_{0}$ and $\mathbf{X}_{1}$, respectively. Define $M$ by

$$
M=\alpha^{-1} \int_{|x|>e}(1+\log \log |x|) \rho(d x) .
$$

Make polar decomposition of $[\rho]_{\{|x|>e\}}$ in such a way that

$$
\rho(E)=-\alpha \int_{[0,2 \pi)} \sigma(d \theta) \int_{e}^{\infty} 1_{E}(r \cos \theta, r \sin \theta) d H_{\theta}(r)
$$

for every Borel set $E$ in $\{x:|x|>e\}$, where $\sigma$ is a probability measure on $[0,2 \pi), H_{\theta}(r)$ is a nonnegative function right-continuous and decreasing in $r \in$ $[e, \infty)$ and Borel measurable in $\theta \in[0,2 \pi)$, and

$$
H_{\theta}(e)+\int_{e}^{\infty} \frac{H_{\theta}(r)}{r \log r} d r=M
$$

that is,

$$
-\int_{e}^{\infty}(1+\log \log r) d H_{\theta}(r)=M
$$

Define

$$
H(r)=\int_{[0,2 \pi)} H_{\theta}(r) \sigma(d \theta)
$$

Then

$$
H(e)+\int_{e}^{\infty} \frac{H(r)}{r \log r} d r=M
$$

Since

$$
I_{0}=\int_{0}^{e^{-1}} \frac{d v}{v} \exp \left[\alpha \int_{v}^{e^{-1}} \frac{d u}{u} \int_{e}^{\infty}\left(1-e^{-u^{\alpha} r}\right) d H(r)\right]
$$

and since the proof of Lemma 4.1 shows that (4.12) holds for any $F_{0}(v)$ satisfying (4.14), the recurrence condition $I_{0}=\infty$ for $\mathbf{X}_{0}$ is equivalent to

$$
\int_{0}^{e^{-1 / \alpha}} \frac{d v}{v} \exp \left[-\int_{e}^{v^{-\alpha}} \frac{H(r)}{r} d r\right]=\infty .
$$

Let 


$$
G(\theta, v)=\alpha \int_{v}^{e^{-1}} \frac{d u}{u} \int_{e}^{\infty}\left(e^{-r u^{\alpha}|\cos \theta+\sin \theta \log u|}-1\right) d H_{\theta}(r)
$$

Then

$$
I_{1}=\int_{0}^{e^{-1}} \frac{d v}{v} \exp \left[-\int_{[0,2 \pi)} G(\theta, v) \sigma(d \theta)\right]
$$

We have

$$
G(\theta, v) \leq \alpha \int_{v}^{e^{-1}} \frac{d u}{u} \int_{e}^{\infty}\left(e^{-2 r u^{\alpha}|\log u|}-1\right) d H_{\theta}(r)
$$

Hence $\int_{[0,2 \pi)} G(\theta, v) \sigma(d \theta)$ also has a bound of the same form with $d H(r)$ in place of $d H_{\theta}(r)$. Now use Lemma 2.2 as in the beginning of the proof of Lemma 5.2. Then we get, as $v \downarrow 0$,

$$
\int_{[0,2 \pi)} G(\theta, v) \sigma(d \theta) \leq \alpha \int_{v}^{e^{-1}} \frac{d u}{u} \int_{e}^{\infty}\left(e^{-r u^{\alpha}|\log u|}-1\right) d H(r)+O(1) .
$$

Since (4.13) holds for any $F_{1}(v)$ satisfying (4.15), we get

$$
\int_{[0,2 \pi)} G(\theta, v) \sigma(d \theta) \leq \int_{e}^{v^{-\alpha}|\log v|^{-1}} \frac{H(r)}{r} d r+O(1),
$$

using (5.14). Notice that

$$
\int_{v^{-\alpha}|\log v|^{-1}}^{v^{-\alpha}} \frac{H(r)}{r} d r \leq H\left(v^{-\alpha}|\log v|^{-1}\right) \log |\log v| \leq \frac{M \log |\log v|}{\log \log \left(v^{-\alpha}|\log v|^{-1}\right)}=O(1) .
$$

Then

$$
\int_{[0,2 \pi)} G(\theta, v) \sigma(d \theta) \leq \int_{e}^{v^{-\alpha}} \frac{H(r)}{r} d r+O(1) .
$$

In order to have estimation from below, fix $\delta$ such that $0<\delta<\pi / 2$ and $\tan \delta$ $<\alpha / 2$ and let

$$
E_{1}=[0, \delta) \cup[\pi, \pi+\delta), \quad E_{2}=[\delta, \pi) \cup[\pi+\delta, 2 \pi) .
$$

Denote $H^{j}(r)=\int_{E_{j}} H_{\theta}(r) \sigma(d \theta)$ for $j=1$,2. If $\theta \in[\pi / 2, \pi) \cup[3 \pi / 2,2 \pi)$ and $u \in\left(0, e^{-1}\right)$, then

$$
|\cos \theta+\sin \theta \log u| \geq|\cos \theta-\sin \theta|=\sqrt{2}\left|\cos \left(\theta+\frac{\pi}{4}\right)\right| \geq 1 .
$$


If $\theta \in[\delta, \pi / 2) \cup[\pi+\delta, 3 \pi / 2)$ and $u \in\left(0, e^{-2 / \sin \delta}\right)$, then

$$
|\cos \theta+\sin \theta \log u| \geq(\sin \delta)|\log u|-1 \geq 1 \text {. }
$$

Hence, if $\theta \in E_{2}$, then

$$
G(\theta, v) \geq \alpha \int_{v}^{e^{-2 / \sin \delta}} \frac{d u}{u} \int_{e}^{\infty}\left(e^{-r u^{\alpha}}-1\right) d H_{\theta}(r)
$$

Therefore

$$
\int_{E_{2}} G(\theta, v) \sigma(d \theta) \geq \alpha \int_{v}^{e^{-2 / \sin \delta}} \frac{d u}{u} \int_{e}^{\infty}\left(e^{-r u^{\alpha}}-1\right) d H^{2}(r) .
$$

Using (4.12) again, we get

$$
\int_{E_{2}} G(\theta, v) \sigma(d \theta) \geq \int_{e}^{v^{-\alpha}} \frac{H^{2}(r)}{r} d r+O(1) .
$$

If $\theta \in E_{1}$, then, letting $\alpha^{-1} \tan \theta=\varepsilon$ and $u^{\alpha}=y$, we have $0 \leq \varepsilon<1 / 2$ and

$$
G(\theta, v) \geq \int_{v^{\alpha}}^{e^{-\alpha}} \frac{d y}{y} \int_{e}^{\infty}\left(e^{-y r(\cos \delta)|1+\varepsilon \log y|}-1\right) d H_{\theta}(r) .
$$

Now let us use Lemma 5.2 here. Then, for $\theta \in E_{1}$,

$$
G(\theta, v) \geq \int_{e}^{v^{-\alpha}} \frac{H_{\theta}(r)}{r} d r-K
$$

with a constant $K$ independent of $\theta$ by virtue of (5.13). Hence

$$
\int_{E_{1}} G(\theta, v) \sigma(d \theta) \geq \int_{e}^{v^{-\alpha}} \frac{H^{1}(r)}{r} d r+O(1) .
$$

Combining (5.17)-(5.19), we see that

$$
\int_{[0,2 \pi)} G(\theta, v) \sigma(d \theta)=\int_{e}^{v^{-\alpha}} \frac{H(r)}{r} d r+O(1)
$$

as $v \downarrow 0$. Consequently it follows from (5.16) that $I_{1}=\infty$ is also equivalent to (5.15). The proof is complete.

\section{REFERENCES}

[1] K. Sato, T. Watanabe, and M. Yamazato, Recurrence conditions for multidimensional processes of Ornstein-Uhlenbeck type, J. Math. Soc. Japan, 46 (1994), 245-265.

[2] K. Sato and M. Yamazato, Stationary processes of Ornstein-Uhlenbeck type, Lec- 
ture Notes in Math. (Springer), 1021 (1983), 541-551.

[3] K. Sato and M. Yamazato, Operator-selfdecomposable distributions as limit distributions of processes of Ornstein-Uhlenbeck type, Stoch. Proc. Appl., 17 (1984), $73-100$.

[4] K. Sato and M. Yamazato, Remarks on recurrence criteria for processes of Ornstein-Uhlenbeck type, Lecture Notes in Math. (Springer), 1540 (1993), $329-340$.

[5] T. Shiga, A recurrence criterion for Markov processes of Ornstein-Uhlenbeck type, Prob. Th. Rel. Fields, 85 (1990), 425-447.

Ken-iti Sato

Department of Mathematics

College of General Education

Nagoya University

Nagoya, 464-01 Japan

Present affiliation

School of Informatics and Sciences

Nagoya University

Nagoya, 464-01 Japan

Toshiro Watanabe

Center for Mathematical Sciences

The University of Aizu

Ikki-machi, Aizu-Wakamatsu

Fukushima, 965 Japan

Kouji Yamamuro

Department of Mathematics

School of Science

Nagoya University

Nagoya, 464-01 Japan

Makoto Yamazato

Department of Mathematics

Nagoya Institute of Technology

Nagoya, 466 Japan

Present affiliation

Departmemt of Mathematics

Ryukyu University

Okinawa, 903-01 Japan 